\title{
Research on Damage Identification of Nonuniform Microcrack in Beam Structures
}

\author{
Jia Guo $(\mathbb{D}$, Deqing Guan $(\mathbb{D}$, and Yanran Pan \\ Department of Civil Engineering, Changsha University of Science \& Technology, Changsha, Hunan, China \\ Correspondence should be addressed to Deqing Guan; 491596452@qq.com
}

Received 30 July 2020; Revised 31 December 2020; Accepted 4 January 2021; Published 18 January 2021

Academic Editor: Carlo Rainieri

Copyright ( 2021 Jia Guo et al. This is an open access article distributed under the Creative Commons Attribution License, which permits unrestricted use, distribution, and reproduction in any medium, provided the original work is properly cited.

\begin{abstract}
Nonuniform microcrack identification is of great significance in mechanical, aerospace, and civil engineering. In this study, the nonuniform crack is simplified as a semielliptical crack, and simplified calculation methods are proposed for damage severity and damage identification of semielliptical cracks. The proposed methods are based on the calculation method for uniform cracks. The wavelet transform and the intelligent algorithm (IA) are used to identify the damage location and the damage severity of the structure, respectively. The singularity of the wavelet coefficient can be used to identify the signal singularity quickly and accurately, and IA efficiently and accurately calculates the structural damage severity. The particle swarm optimization (PSO) algorithm and the genetic algorithm (GA), widely used, are applied to identify the damage severity of the beam. Numerical simulations and experimental analyses of beams with transfixion and semielliptical cracks are carried out to evaluate the accuracy of the semielliptical crack calculation method and the method of wavelet analysis combined with PSO and GA for nonuniform crack identification. The results show that the wavelet-particle swarm optimization (WPSO) and the wavelet-genetic algorithm (WGA) can accurately and efficiently identify the structural semielliptical damage location and severity and that these methods are not easily influenced by noise. The damage severity calculation method for semielliptical cracks can accurately calculate the semielliptical size and can be used to identify damage in beams with semielliptical cracks.
\end{abstract}

\section{Introduction}

In the field of aerospace, mechanical engineering, and civil engineering, the damaged component will appear fatigue failure under long-term load, which has a huge impact on safety of structure. Therefore, the structural damage identification and life prediction are of great significance and importance. In the long-term service of steel structure, the notch is caused by the welding defect or the external force, and the crack is one of the most common form of damages. The form of crack is a problem that cannot be ignored in damage identification. Only when the size of a crack in a certain direction is large, can the damage in that direction be approximately reduced to a uniform crack, while for the nonuniform damage, there will be a big error in simplifying it to a uniform crack. At present, the related research studies mainly focus on the damage identification of uniform cracks [1-5] but few on the damage identification of nonuniform cracks and nonuniform propagation [6]. The author has not found the calculation equation for the damage identification of semielliptical cracks at present. Therefore, the damage identification of beams with nonuniform microcracks is studied, and the damage calculation method of the beam with semielliptical cracks is proposed.

In the past decades, a large number of experts and scholars have made great efforts to develop nondestructive identification technology to identify structural damage. The nondestructive identification method based on structural vibration is widely used. Experts and scholars in relevant fields have used the vibration response as the basis for damage detection in the past decade $[7,8]$ and proposed some relevant methods $[9,10]$. In 1979, Cawley and Adams [11] applied natural frequency to damage identification. Although the method based on natural frequency has the advantages of fast and easy implementation, it has found many disadvantages with the development of research, such 
as insensitive to microdamage, vulnerable to noise, temperature, and humidity, low sensitivity to local damage, and difficult to accurately locate and quantify damage [12-14]. Modal shapes can be used as an index to identify structural damage with characteristics of insensitivity to temperature, humidity, and other environmental conditions. The displacement modal shape, as a global parameter, is less sensitive to local damage, which makes it difficult to accurately locate the damage and quantify the damage. The strain mode has more information than the displacement mode and can be used as an index to accurately locate the damage location. The modal shape of the nondestructive structure should be smooth and continuous [15]. The structural damage will be accompanied by the reduction of the local stiffness, and the modal shape of the damaged area will no longer be continuous and smooth. The detection of such discontinuity is the main target of the vibration-based damage detection method.

Wavelet transform is widely used in the field of structural health detection and damage detection because of its good denoising ability and singularity detection for signal. Wavelet transform can find singular points from the modal shape of the structure without prior knowledge of the damage area, which makes the singularity of wavelet coefficient become an excellent index of the damage location of the structure and can display the damage location intuitively. Therefore, the method of detecting the discontinuity of structural modal shape based on wavelet singularity has attracted the attention of researchers. Some researchers have applied WT to decompose the structural acceleration and modal shape to identify the damage $[5,16]$. For example, Zhu and Law [17] presented a crack identification technique for bridge beam structures under the moving load and analyzed the displacement mode of the bridge structure through continuous wavelet transform (CWT) to identify the crack damage location of the bridge structure. He et al. [18] furthered this by applying moving load to excite the bridge structure to obtain displacement response and applied discrete wavelet transform (DWT) to decompose the displacement response to locate the potential damage location. Zhou and Li [19] proposed a damage identification method based on the curvature mode and two-dimensional continuous wavelet transform, which is utilized to identify the damage of composite sandwich panels. The curvature mode is processed by two-dimensional continuous wavelet transform, and the damage index is constructed by the periodicity and wavelet transform coefficient of the structure to identify the structural damage. Xu et al. [6] improved the two-dimensional modal curvature with complex wavelet transform and obtained the two-dimensional modal curvature of wavelet. The identification ability of the two-dimensional modal curvature of complex wavelet was analyzed and verified by experiments through the plate with nonuniform cracks, and the applicability of the method was further discussed by taking the plate sluice with nonuniform cracks as an example. The results show that the two-dimensional mode curvature of complex wavelet can characterize the length, degree, and nonuniformity of nonuniform cracks, which provides a suitable tool for the identification and characterization of plate structure cracks under noisy conditions. Although wavelet transform based on vibration can effectively identify the structural damage location, the microdamage severity identification method based on wavelet is not as reliable as the damage location identification method based on wavelet, especially in the case of errors in the measurement of structural vibration data. Therefore, IA is applied to identify the structural damage severity in this study.

IA is widely used in many subjects with the characteristics of simple, universal, and convenient for parallel processing and so on. The common method of applying IA to structural damage identification is to describe the problem of damage identification as an optimization problem of objective function. The idea is the difference between the calculation results of the finite element model and the structural experiment data is defined as the objective function. When the objective function reaches the global minimum value, the input parameters of the finite element model are taken as the physical parameters of the actual structure so that the structural damage identification problem is transformed into the extreme value problem of the corresponding objective function. Finally, IA is applied to quickly and accurately calculate the optimal solution of the objective function in the solution space. Some experts and scholars have already applied the intelligent optimization algorithm to structural damage identification. Hao and Xia [20] applied GA to search the minimum value of the objective function and identified the structural damage location by considering the change of frequency and mode shape as the damage parameters. Sahu and Nayak [21] took the natural frequency of the structure as parameters and introduced a method of data filtering in GA to filter the data so as to obtain the rigidity of the structure. This method improved the adaptability of GA to data pool and solution space and improved the calculation accuracy of GA. Begambre and Laier [22] proposed the particle swarm optimization-simplex (PSOS) algorithm to identify structural damage, and compared with the simulated annealing and the basic PSO, it is proved that the PSOS algorithm has better performance in identifying the structural damage location and severity. Nanda et al. [23] proposed particle swarm optimization with incremental swarm size (I-PSO) technique to identify the location and quantity of cracks in beam structures. The minimum value of objective function is calculated by I-PSO to identify the damage of structure, and the numerical simulation analysis shows that I-PSO can effectively identify the damage of the structure and is robust to noise. Guedria [24] defined the damage identification of plate structure as a nonlinear optimization problem and calculated the minimum value of the constructed objective function with the accelerated differential evolution (ADE) algorithm so as to accurately identify the damage location and severity of plate structure. Compared with the standard difference evolution algorithm, ADE has three improvements, which make ADE have the advantages of fast convergence, automatic balance of global and local search ability, and avoiding premature convergence of local optimal solution. The numerical simulation results show that $\mathrm{ADE}$ can effectively identify structural damage and is difficult to be influenced by noise. Moradi et al. [25] applied the bees 
algorithm to identify the cracks in the beam structures and predicted a single open edge crack in the cantilever beam through numerical simulation and experimental research. The results show that the method can predict the size and location of the crack well. Guo and Li [26] proposed a twostep damage identification method based on Bayesian theory and improved the immune genetic algorithm. Firstly, Bayesian theory is utilized to integrate the two information sources of structural modal strain energy and frequency to identify the location of structural damage, and then the immune genetic algorithm is used to identify the severity of structural damage. The simulation results show that the twostep method can accurately identify the structural damage location and severity, and the improved genetic algorithm is better than the simple genetic algorithm and optimal strategy genetic algorithm. $\mathrm{Yu}$ and $\mathrm{Xu}$ [27] transformed the structural damage identification problem into a constraint optimization problem, which was solved by the ant colony optimization-based algorithm. Through the numerical simulation and the experiment of a 3-story building rigid frame, the results show that regardless of weak damage or multiple damages, the identification accuracy is very high and the noise immunity is better. Zhao et al. [28] combined the tabu search method and chaos search method to optimize the artificial bee colony algorithm and improved the exploration and development ability of the algorithm. The numerical simulation and experiment proved that the proposed method can effectively identify structural damage and has good robustness under the condition of sufficient measurement data.

IA can accurately identify the structural damage location and severity, but the parts of the structure without damage will also be calculated by IA, which greatly affect the identification efficiency of IA. Therefore, to improve the identification efficiency, it is worth studying that the damage location should be located firstly and then the damage severity of the damage location should be calculated by IA. At present, there are many methods to identify structural damage with two steps, most of which combine with IA to identify structural damage are using IA to identify the damage severity. Xiang and Liang [1] proposed a two-step approach for detecting multiple damages in thin plates. In the first step, the mode shape of two-dimensional wavelet transform was used to reveal the singularity and hence the damage locations. The damage severities at the identified locations are then assessed in the second step by PSO. The simulation studies of a simply supported plate with multiple damages show that the proposed method is effective in multidamage detection and performed reasonably well even the natural frequencies are not precisely measured, and the analysis shows that the higher natural frequency is, the more accurate results of damage severity identification will be. Ravanfar et al. [2,29] defined the structural damage index by wavelet packet entropy in the structural damage identification of beams to identify the damage location, and then GA was utilized to calculate the damage severity of the damage location. The influence of different noise level, damage condition, wavelet type, and decomposition level on damage location recognition was studied. The results of numerical simulation and experimental analysis show that the combination of wavelet and GA can effectively and accurately identify the structural damage location and severity and can be applied to the damage identification of the engineering structure. In this study, the inverse problem method is used to calculate the damage severity, and the IA is used to calculate the objective function set in the program as the inverse problem calculation objective. The structural damage severity can be obtained by calculating the minimum value of the objective function. Nanthakumar et al. [30], according to the principle of solving inverse problem, employed a collocation formulation for solving $2^{\text {nd }}$ order boundary value problems. Anitescu et al. [31] proposed an algorithm to solve the inverse problem of detecting inclusion interfaces in a piezoelectric structure and gave the calculation formula of inclusions with three-dimensional structure and different materials. The numerical example results proved that the proposed iterative method can determine the location and approximate shape of material subdomains under high noise conditions.

The singularity of wavelet coefficient is intuitive and concise, and the IA has the advantages of high efficiency and reliability. The combination of wavelet analysis and IA can effectively synthesize the advantages of both so as to maximize the efficiency of structural damage identification. Compared with other IAs, PSO and GA are widely applied in structural damage identification [32-37]. In this study, the microdamage severity is defined to be within $10 \%$. Because the author is restricted by the experimental equipment, the microdamage only refers to the damage in the depth direction of the damage form, and the width of the damaged form is the same as the width of the high sensitivity vibration sensor used in the experiment. In this study, the beam structural damage width is not a parameter to be considered in the calculation of damage severity, so the width of damaged form has no effect on the structural damage identification. The proposed calculation method and identification method of beam structural semielliptical damage can be verified by notch damage identification. After the structural modal data were obtained, the wavelet transform is combined with PSO and GA, respectively, to process the modal data so as to identify the structural microdamage.

Whether the structure has boundary damages or not, the wavelet coefficients obtained by WT decomposition signal will have boundary distortion which will lead to the distortion of WT identification of boundary damage. At present, the existing solutions to boundary distortion are signal expansion [38,39], zero compensation $[40,41]$, extrapolation signal expansion [42], truncation boundary signal, [8] and so on. These methods are based on the method of distorting the original signal to avoid boundary distortion, which will lead to the increase in computational complexity and the loss of information, resulting in the unreliable damage identification. When the damage occurs at the boundary, the above methods cannot solve the problem of boundary damage identification. Therefore, this study will not consider the boundary microdamage of the structure only consider the location where the damage exists between $1 / 5$ and $4 / 5$ of the length of the beam. 
There are currently damage identification methods for nonuniform cracks, but the author has not identified any specific calculation method for the damage size of nonuniform cracks. To calculate the damage size of a nonuniform crack, it can be simplified into a semielliptical crack. The specifics of this simplified method are described in Section 2. In this study, the damage severity of transfixion cracks is calculated firstly, and the damage severity of beams with semielliptical surface cracks is defined using the same moment of inertia (assuming that the damage severity is the same). The wavelet is combined with PSO and GA to identify the damage severity of beams with nonuniform cracks, respectively, and the semielliptical crack size is calculated based on the identified damage severity. The validity and accuracy of the crack damage identification method (based on the wavelet-intelligent algorithm) are verified by notch damage identification. The calculation method for the damage size of beams with semielliptical cracks is verified by numerical simulations and experiments; the results show that the proposed method is of high accuracy and can be used effectively for semielliptical cracks calculations in engineering structures.

\section{Calculation Model of Semielliptical Crack}

In this study, a the uniform crack is defined as having a height that does not change along the thickness direction of the beam, and the nonuniform crack is defined as having a height that changes along the thickness dimension of the beam. The uniform crack in beam structures is a crack with equal height transfixion along the thickness direction, and the nonuniform crack in beam structures is semielliptical crack along the thickness direction. These two types of cracks are the object used to explain the damage identification method for the nonuniform crack.

In fracture mechanics, the definition of crack not only refers to the cracks caused by various factors but also the micro defects existing in the materials, such as pores, knife marks, and bubbles produced in smelting, which are called cracks. In the view of mechanics, cracks can be divided into open, sliding, and tearing types, as shown in Figure 1 [43].

In the current damage research, cracks can be treated as two categories: transfixion cracks and nontransfixion cracks. It is an ideal way to simplify the crack as a transfixion crack, but in practical engineering, the crack in the structure can be approximately simplified as a transfixion crack only when the crack in a certain direction is large in size. In general, the nontransfixion cracks are more suitable for practical application, which makes the nontransfixion cracks not to be ignored in structural damage identification. Nontransfixion cracks are classified as embedded cracks and surface cracks.

The embedded crack refers to the structural internal defect, and the size of the crack is small relative to the size of the component. According to the law of crack propagation, the embedded crack can be simplified as elliptical crack, as shown in Figure 2.
The long axis of the ellipse is $2 c$, and the short axis is $2 a$. The coordinate $\left(x_{p}, y_{p}\right)$ of point $p$ on the crack ellipse boundary satisfies the elliptic equation:

$$
\frac{x_{p}^{2}}{c^{2}}+\frac{y_{p}^{2}}{a^{2}}=1
$$

which is expressed by parameter $\theta$ as follows:

$$
\left\{\begin{array}{l}
x_{p}=c \cos \theta \\
y_{p}=a \sin \theta
\end{array}\right.
$$

The surface crack is located on the structural surface and is often simplified as the semielliptical sheet crack [44], as shown in Figure 3. In practical engineering, there are more structures with semielliptical surface cracks. Therefore, this paper will study the calculation formula and damage identification of the damage severity and damage size of the beam structure with semielliptical crack damage.

In this section, the relationship between the damage severity and the rigidity of the structure with transfixion cracks is defined first, and then the damage severity of the semielliptical surface crack is defined. Finally, the relationship between the damage severity of semielliptic surface crack and the crack depth $a$ is defined by fitting the functional relationship between section size $b, h$, and $a$.

2.1. Transfixion Cracks. The stiffness reduction method is used to simulate the damage of the beam with transfixion cracks, and the following assumptions are made:

(1) The damage is linear without considering the crack opening and closing.

(2) The structural damage is expressed as the decrease in stiffness.

(3) The damage does not affect the structural stability.

(4) The damage occurs in the interior of the structure, which only affects the eigenvalue and mode shape of the structure, and the boundary conditions remain unchanged.

(5) The crack size is relatively small compared with the beam, so the influence of the height change of the beam is ignored, and the change of the central axis of the beam caused by the crack is not considered.

Figure 4 shows a beam with transfixion crack. $h$ is the section height, $b$ is the width, $x_{d}$ is the distance between the crack location and the left end of the beam, $\Delta l$ is the crack width, and $h_{d}$ is the crack depth.

The section moment of inertia $I_{d}$ at the crack is as follows:

$$
I_{d}=\frac{1}{12} b\left(h-h_{d}\right)^{3}=\frac{1}{12} b h^{3}\left(1-\frac{h_{d}}{h}\right)^{3}=I_{0}\left(1-\frac{h_{d}}{h}\right)^{3},
$$

where $I_{0}$ is the moment of inertia of the undamaged section. Definition $\varepsilon=\left(h_{d} / h\right)$ is damage severity, then equation (3) becomes the following: 


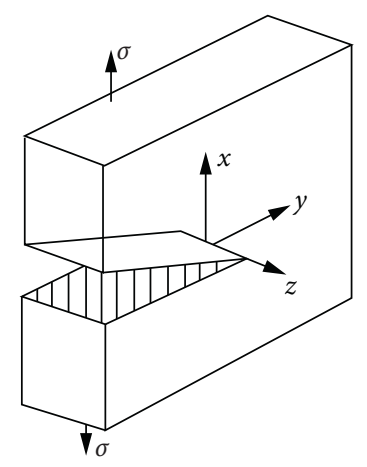

(a)

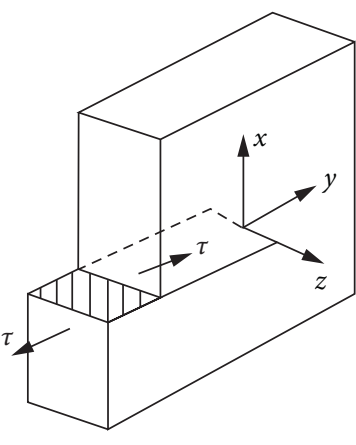

(b)

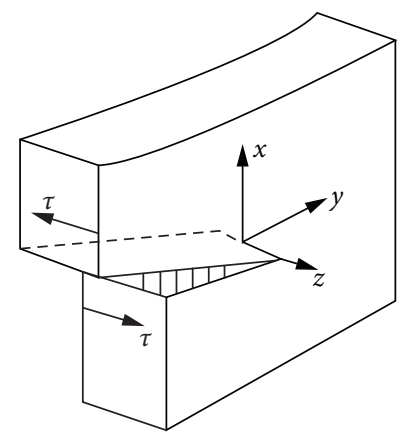

(c)

FIgURE 1: Classification of mechanical characteristics of cracks: (a) opening type; (b) sliding type; (c) tearing type.

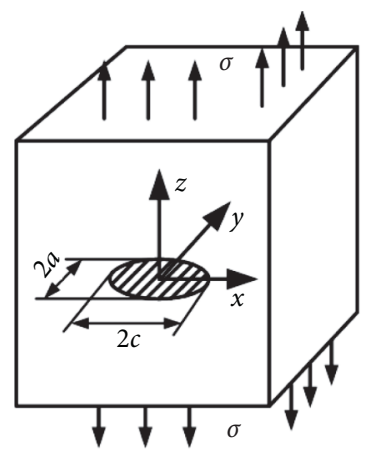

(a)

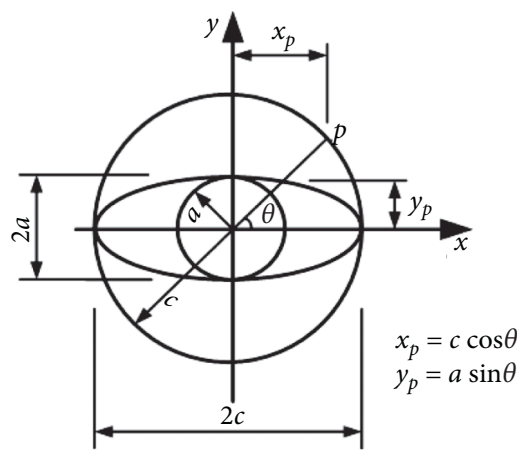

(b)

FIGURE 2: Embedded crack of elliptical.

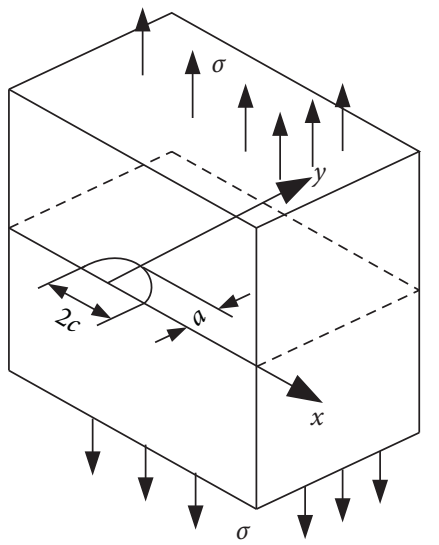

(a)

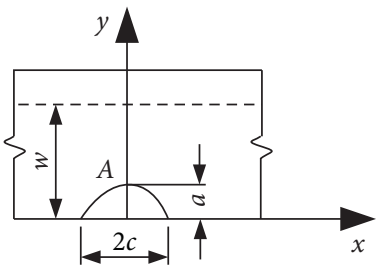

(b)

Figure 3: Semielliptical surface crack.

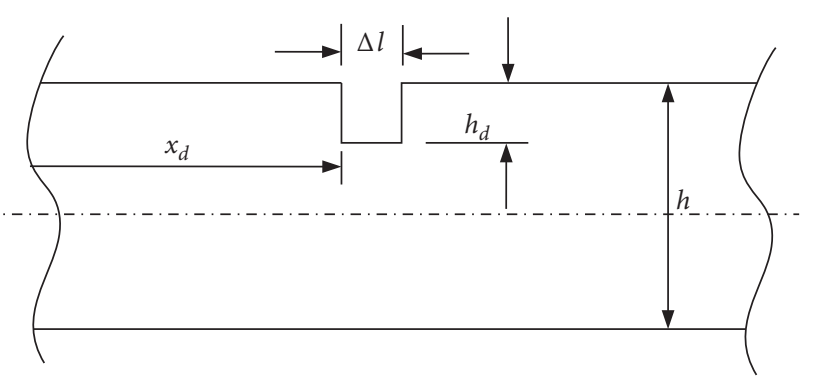

Figure 4: Beam with transfixion crack. 


$$
I_{d}=I_{0}(1-\varepsilon)^{3} .
$$

2.2. Semielliptical Cracks. The beam with symmetrical nontransfixion semielliptical surface cracks is shown in Figure 5, where $c$ s the half length of crack (semielliptical semimajor axis) and $a$ is the crack depth (semielliptical semiminor axis).

The semielliptical crack area $A$ of the damaged beam section can be expressed as follows:

$$
A=\frac{1}{2} \pi a c \text {. }
$$

Equation (5) shows that $a$ and $c$ are variables related to the semielliptical crack area. According to the law of crack propagation, the deeper $a$ of the semielliptical crack is, the longer $2 c$ of the crack length will be. Morozov [45] proposed a graphic analysis method to calculate the critical size (depth and length) of semielliptical cracks. The American Society of Mechanical Engineers considers that the ratio of crack depth (semielliptical semiminor axis) $a$ to semielliptical semimajor axis $c$ is constant. The British Standard "Guidelines for several methods of acceptance criteria for welding defects" holds that the crack length $c$ remains unchanged until the crack shape becomes circular and the circle crack remains at the end of the crack. Newman and Raju [46] controlled the ratio range between crack depth $a$ and crack length $c$ when studying the empirical equation of crack stress intensity factor, and the proposed classical crack shape hypothesis method has been widely used to solve the surface crack problem of crack depth $a$. In the steady state of crack propagation, Ibso and Agerskov [44] take the ratio of crack depth to crack half length $a / c=1 / 4$ as the fixed value in crack research. Based on the above discussion, this paper refers to the study of Ibso and assumes that the area of a semiellipse as follows:

$$
A=\frac{1}{2} \pi a c=2 \pi a^{2} .
$$

Equation (6) shows that the crack depth becomes the only variable to change the section moment of inertia when the section size is constant.

The damage of section will cause the change of stiffness, which is caused by the decrease in the moment of inertia without considering the change of the elastic modulus of the material. When the moment of inertia of the section with semielliptical surface crack in the same section of the beam is the same as that of the section with transfixion crack, the damage severity will be the same.

Definition 1. $I_{\text {sd }}$ is the moment of inertia of the damaged section of the beam with a semielliptical surface crack, and $\varepsilon_{s}$ is the damage severity of the beam with a semielliptical surface crack. Based on the above discussion, the damage severity of the section with semielliptical surface crack and

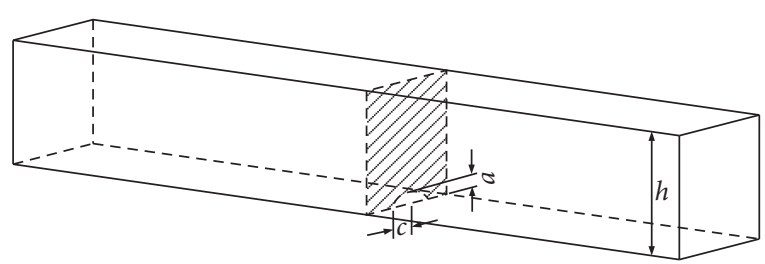

Figure 5: Beam with semielliptical crack.

the section with transfixion crack in the beam is treated equivalently; that is, $\varepsilon_{s}=\varepsilon$ when $I_{\text {sd }}=I_{d}$. It is very complicated to calculate the principal moment of inertia for the damaged section with a semielliptic surface crack, so the main moment of inertia of the damaged section is obtained by CAD software in this study.

The damage severity $\varepsilon$ can be defined by the decrease in the transfixion crack height $h$, while the damage severity cannot be defined directly by the semielliptical surface crack height $h$. In this paper, the damage severity is equivalent with the moment of inertia of the damage section; the functional relationship among the section size, the crack depth (the semiminor axis of the semiellipse) $a$, and the damage severity $\varepsilon$ is established, and the damage severity $\varepsilon_{s}$ of the structure with the semielliptical surface crack is corresponding to the damage severity $\varepsilon$ of the structure with the transfixion crack.

Taking the simple supported beam model with the section size of $60 \mathrm{~mm} \times 80 \mathrm{~mm}$ as an example, the relationship between crack depth $a$ and damage severity $\varepsilon_{0}$ of the semielliptical surface crack was found by selecting 10 cross sections with different crack depths. The fitting curve is shown in Figure 6.

The expression of the curve in Figure 6 is as follows:

$$
\varepsilon_{0}=-0.0066+\frac{2.87731}{10.97251 \sqrt{\pi / 2}} \exp \left[-2\left(\frac{a-15.14937}{10.97521}\right)^{2}\right]
$$

In addition, when the crack depth is the same and the section size is different, the damage severity will change. Eight simple supported beams with different section sizes $b$ and $h$ are taken as the research objects. When the crack depth is the same, the damage severity $\varepsilon_{1}$ of each beam is regarded as a group of data. As shown in Table 1, two groups of data with different crack depth are selected to fit the relationship between the section area ratio $(b h / 60 \times 80)$ and the damage severity ratio $\left(\varepsilon_{1} / \varepsilon_{0}\right)$. The calculation results are shown in Table 1 , and the average value of $\left(\varepsilon_{1} / \varepsilon_{0}\right)$ of different $a$ values is shown in Table 2 so as to fit the calculation formula:

$$
\frac{\varepsilon_{1}}{\varepsilon_{0}}=0.9999\left(\frac{b h}{60 \times 80}\right)^{-1.009}
$$

Substituting equation (7) into equation (8), we can obtain the following: 


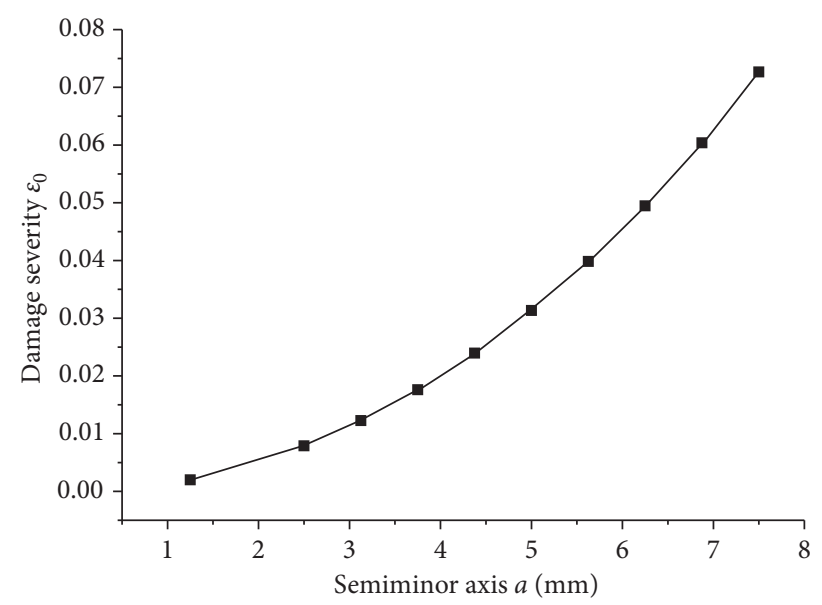

FIgURE 6: Relationship between $a$ and $\varepsilon_{0}$.

TABLE 1: The relation between cross section area and damage severity.

\begin{tabular}{|c|c|c|c|c|c|c|}
\hline$a(\mathrm{~mm})$ & $B(\mathrm{~mm})$ & $h(\mathrm{~mm})$ & $\varepsilon_{1}$ & $\varepsilon_{0}$ & $(b h /(60 \times 80))$ & $\left(\varepsilon_{1} / \varepsilon_{0}\right)$ \\
\hline \multirow{8}{*}{3.75} & 30 & 50 & 0.058302 & \multirow{8}{*}{0.0175683} & 0.3125 & 3.312597 \\
\hline & 35 & 50 & 0.049067 & & 0.364583333 & 2.787919 \\
\hline & 40 & 60 & 0.035565 & & 0.5 & 2.020727 \\
\hline & 45 & 60 & 0.031346 & & 0.5625 & 1.781001 \\
\hline & 50 & 70 & 0.024198 & & 0.729166667 & 1.374886 \\
\hline & 55 & 70 & 0.021896 & & 0.802083333 & 1.244074 \\
\hline & 60 & 80 & 0.01761 & & 1 & 1.000565 \\
\hline & 65 & 80 & 0.016209 & & 1.083333333 & 0.92097 \\
\hline \multirow{8}{*}{1.25} & 30 & 50 & 0.006353 & \multirow{8}{*}{0.0018519} & 0.3125 & 3.17644 \\
\hline & 35 & 50 & 0.005435 & & 0.364583333 & 2.717603 \\
\hline & 40 & 60 & 0.00398 & & 0.5 & 1.989873 \\
\hline & 45 & 60 & 0.003534 & & 0.5625 & 1.767181 \\
\hline & 50 & 70 & 0.002736 & & 0.729166667 & 1.367993 \\
\hline & 55 & 70 & 0.002486 & & 0.802083333 & 1.243001 \\
\hline & 60 & 80 & 0.002 & & 1 & 0.999815 \\
\hline & 65 & 80 & 0.001845 & & 1.083333333 & 0.922619 \\
\hline
\end{tabular}

TABLE 2: Average calculation results of $\varepsilon_{1} / \varepsilon_{0}(a=1.25 \mathrm{~mm}$ and $3.75 \mathrm{~mm})$.

\begin{tabular}{lcc}
\hline$\left(\varepsilon_{1} / \varepsilon_{0}\right)(a=3.75 \mathrm{~mm})$ & $\left(\varepsilon_{1} / \varepsilon_{0}\right)(a=1.25 \mathrm{~mm})$ & Average value of $\left(\varepsilon_{1} / \varepsilon_{0}\right)$ \\
\hline 3.312597 & 3.17644 & 3.244518177 \\
2.787919 & 2.717603 & 2.752760992 \\
2.020727 & 1.989873 & 2.00530003 \\
1.781001 & 1.767181 & 1.774090595 \\
1.374886 & 1.367993 & 1.371439421 \\
1.244074 & 1.243001 & 1.243537162 \\
1.000565 & 0.999815 & 1.000190063 \\
0.92097 & 0.922619 & 0.921794422 \\
\hline
\end{tabular}

$$
\varepsilon_{1}=\frac{5179.9907}{(b h)^{1.009}}\left\{-0.0066+\frac{2.87731}{10.97251 \sqrt{\pi / 2}} \exp \left[-2\left(\frac{a-15.14937}{10.97521}\right)^{2}\right]\right\}
$$


The significance of equation (9) is that it provides a method to calculate the size of nonuniform cracks for structures with nonuniform cracks. After identifying the structural nonuniform cracks, the approximate size of the nonuniform cracks can be quickly calculated by equation (9), which can provide specific data for the follow-up steps of structural health monitoring.

\section{Theoretical Background}

3.1. The Principle of Wavelet Singularity. Defining $\psi(t) \in L^{1}(R) \cap L^{2}(R)$ and $\int_{-\infty}^{+\infty} \psi(t) \mathrm{d} t=0, \psi(t)$ is called a basic wavelet or a mother wavelet. $\psi(t)$ is scaled and translated as follows:

$$
\psi_{a, b}(t)=\frac{1}{\sqrt{|a|}} \psi\left(\frac{t-b}{a}\right), \quad a, b \in R, a \neq 0,
$$

where $a$ and $b$ are the scale factor and translation factor, respectively, and $\psi_{a, b}(t)$ is the wavelet function.

Defining $\psi(t)$ as the basic wavelet, the continuous wavelet transform (CWT) of $f(t) \in L^{2}(R)$ is defined as follows:

$$
W f(a, b)=\frac{1}{\sqrt{|a|}} \int_{-\infty}^{+\infty} f(t) \psi^{*}\left(\frac{t-b}{a}\right) \mathrm{d} t=\left\langle f, \psi_{a, b}\right\rangle,
$$

where $a \neq 0$, band $t$ are continuous variable, and $\psi^{*}(t)$ is the complex conjugate of $\psi(t)$.

The convolution form of wavelet transform is as follows:

$$
W f(a, b)=\frac{1}{\sqrt{|a|}} \int_{-\infty}^{+\infty} f(t) \psi^{*}\left(\frac{t-b}{a}\right) \mathrm{d} t=\sqrt{|a|} f * \widehat{\psi}_{|a|}(b),
$$

where $\widehat{\psi}_{|a|}(t)=|a|^{-1} \psi^{*}(-t / a)$.

Defining $\theta(t)$ as a smooth function and derivative $\psi(t)=(d \theta(t) / \mathrm{d} t)$ of any low-pass function $\theta(t)$ is bandpass function, which satisfies $\int_{-\infty}^{+\infty} \psi(t) \mathrm{d} t=0$, the wavelet function with scale factor $s$ is as follows:

$$
\psi_{s}(t)=\frac{1}{s} \psi\left(\begin{array}{l}
t \\
s
\end{array}\right)=\frac{1}{s} \frac{d \theta(t / s)}{\mathrm{d} t}=\frac{d \theta_{s}(t)}{\mathrm{d} t} .
$$

The continuous wavelet transform of the basic wavelet corresponding to function $f$ on $s$ is as follows :

$$
W f(s, u)=s^{1 / 2}\left(f * \bar{\psi}_{s}\right)(u)=s^{1 / 2} \frac{d}{\mathrm{~d} u}\left(f * \bar{\theta}_{s}\right)(u),
$$

where $s>0$, and equation (14) shows that the modulus maximum $|W f(s, u)|$ of the wavelet transform is the maximum of the first derivative of $f$ smoothed by $\bar{\theta}_{s}$ and corresponds to the singular point of signal.

3.2. Damage Location Identification. At the moment of damage, the mass of the beam structure with microdamage is basically the same, so it can be considered that the structural damage is essentially the reduction of stiffness EI. The stiffness of both sides of the damaged location is not equal, i.e., $\mathrm{EI}\left(v^{+}\right) \neq \mathrm{EI}\left(v^{-}\right)$. When $v=x$, the deformation condition and internal force balance condition of the position still meet the following conditions [47]:

Vertical displacement:

$$
w\left(v^{+}\right)=w\left(v^{-}\right)
$$

Rotation angle:

$$
\frac{d w\left(v^{+}\right)}{\mathrm{d} x}=\frac{d w\left(v^{-}\right)}{\mathrm{d} x}
$$

Bending moment:

$$
\mathrm{EI}\left(v^{+}\right) \frac{d^{2} w\left(v^{+}\right)}{\mathrm{d} x^{2}}=\mathrm{EI}\left(v^{-}\right) \frac{d^{2} w\left(v^{-}\right)}{\mathrm{d} x^{2}} .
$$

Shearing force:

$$
\mathrm{EI}\left(v^{+}\right) \frac{d^{3} w\left(v^{+}\right)}{\mathrm{d} x^{3}}=\mathrm{EI}\left(v^{-}\right) \frac{d^{3} w\left(v^{-}\right)}{\mathrm{d} x^{3}},
$$

where the superscripts + and - are used to denote the quantities just at the right and left of the discontinuous point. $k$ is the curvature of the section, $\rho(x)$ is the radius of curvature, $M$ is the bending moment, $\varepsilon$ is the strain, $\mu$ is the severity of deformation, and $h$ is the distance from the point on the section to the neutral layer.

According to equations (17) and (18), microdamage will also cause the curvature mode and strain mode on both sides of the damage location to be unequal. In addition, the curvature mode and strain mode far away from the damage location of the structure will not change greatly, so the curvature mode and strain mode of the structure will mutate at the damage location. Previous research studies show that the strain mode is more sensitive to the microdamage of the structure, so the strain mode of the damaged structure will be analyzed by the continuous wavelet transform in this study, and the singular point (modulus maximum point) of the structural strain mode signal will be used to identify the damage location of the structure.

3.3. Strain Mode. Strain is the first derivative of displacement, so each displacement mode corresponds to the corresponding strain mode, and the strain mode reflects the structural inherent characteristics. Because the strain modes cannot be measured directly, the curvature mode can be used to measure the strain modes indirectly. According to the material mechanics, the bending static relation of the beam can be obtained as follows:

$$
\rho_{i}=\frac{1}{d_{i}}=\frac{M_{i}}{E_{i} I_{i}}
$$

where $i$ is the section position of measuring point $i, M_{i}$ is the bending moment of section $i, E_{i} I_{i}$ is the flexural rigidity of section $i, d_{i}$ is the radius of curvature at section $i$, and $\rho_{i}$ is the curvature of section $i$. According to the approximate equation of bending deformation of the beam, 


$$
\rho=\frac{d^{2} y}{\mathrm{~d} x^{2}}
$$

where $x$ is the coordinate along the length direction of the straight beam and $y$ is the bending deflection of the beam. According to equations (19) and (20), the difference equation of three equidistant continuous measuring points along the beam can be obtained:

$$
\rho_{i}=\frac{M_{i}}{E_{i} I_{i}}=\frac{y_{i+1}-2 y_{i}+y_{i-1}}{\Delta 2},
$$

where $i-1, i$, and $i+1$ are three adjacent continuous measuring points with equal distance along the beam, $\rho_{i}$ is the curvature of section $i, y_{i}$ is the bending deflection of section $i, y_{i+1}$ and $y_{i-1}$ are the bending deflection of section $i-1$ and section $i+1$ of the measuring points, respectively, and $\Delta$ is the bending deflection of two adjacent measuring points. The strain $\varepsilon_{i}$ of the measuring point $i$ of the beam can be expressed as follows:

$$
\varepsilon_{i}=-\frac{h}{d_{i}}=-h \frac{y_{i+1}-2 y_{i}+y_{i-1}}{\Delta^{2}}=-h \rho_{i},
$$

where $h$ is the distance between the surface of the measuring point on the beam and the neutral layer, and equation (22) shows that the strain mode is the second-order difference or second derivative of the displacement mode.

3.4. Principle of PSO. PSO is a population-based stochastic optimization technology, which has the advantages of simple to use, fast convergence, and good robustness. The basic idea of PSO is to find the optimal solution through the cooperation and information sharing among individuals in the group. When PSO is used to solve the optimization problem, finite possible solutions of the optimization problem can be initialized to the particle swarm, and the individual fitness and average fitness of the whole population will be continuously improved by the iterative process. When the optimal solution of the problem is obtained or the optimal particle position in the solution space is found, the individual fitness of the particles in the group no longer changes significantly, and the population will stop iteration and output the optimal solution of the problem at the same time.

The PSO's process is as follows:

(1) Establishing the unknown function: The method of the element stiffness reduction is used to simulate the damage severity $\gamma_{i}$ of the structural damage element, and the unknown value $\gamma$ of objective function is constructed by taking $\gamma_{i}$ of all structural damage elements to be identified as an unknown component. If the stiffness of the damaged element isEI, the damage severity is $\gamma$, and if the stiffness of the damaged element is $\mathrm{EI}_{i}$, then

$$
\mathrm{EI}_{i}=(1-\gamma) \mathrm{EI} \text {. }
$$

Since the damage is set as microdamage, it is assumed that the mass and elastic modulus $E$ of the damaged element do not change before and after the structural damage, only the size of the element changes before and after the structural damage.

(2) Constructing the objective function: In this study, based on the comprehensive consideration of the global and local attributes of the structure, frequency and displacement mode are adopted as the calculation parameters of the objective function, as shown in the following equation:

$$
\begin{aligned}
\min F= & F_{\omega} \sum_{i=1}^{m}\left(\frac{f_{i}^{\mathrm{test}}-f_{i}^{\mathrm{cal}}(\gamma)}{f_{i}^{\mathrm{test}}}\right)^{2} \\
& +F_{\phi} \sum_{i=1}^{n} \sum_{j=1}^{k}\left(\phi_{i j}^{\mathrm{test}}-\phi_{i j}^{\mathrm{cal}}(\gamma)\right)^{2}
\end{aligned}
$$

where the weighting factor $F_{w}=F_{\varphi}=1 . f_{i}^{\text {test }}$ and $f_{i}^{\text {cal }}$ are the $i$-th order natural frequencies measured and calculated, respectively, $\phi_{i j}^{\text {test }}$ and $\phi_{i j}^{\text {cal }}$ are the displacement modes of the $i$-th order $j$-th node measured and calculated after normalization, respectively, $m$ is the order of natural frequency, $n$ is the order of the displacement mode, and $k$ is the node number. The unknown value $\gamma$, which minimizes the function $\min F$, is the set of damage severity $\gamma_{i}$ of the structural all damaged elements.

(3) Establishing fitness function: The objective function is directly used as the fitness function of PSO to identify the structural damage severity, that is, $\mathrm{FIT} 1=\min F$.

(4) Establishing PSO and solving the fitness function.

3.5. Principle of GA. GA has strong global search ability and is not easy to fall into local optimum. When GA is used to solve the optimization problem, the possible solution of the optimization problem can be regarded as an individual in a certain population, then all the possible solutions in the solution space constitute an initial population. When the initial population is placed in the optimization problem, the individuals in the group can judge the advantages and disadvantages of their own chromosomes through the fitness function, and then the individual chromosomes are selected that can better adapt to the optimization problem according to the principle of survival of the fittest. Finally, the selected individuals produce a new generation of individuals with higher adaptability to the optimization problem through genetic evolution such as crossover and mutation. By repeating the above genetic evolution mechanism, the population can improve its own fitness and the average fitness of the whole population. When the fitness of the individuals in the population does not change significantly or the optimal individual within the population has been found, the whole population will stop genetic evolution and output the optimal individuals within the current population. 


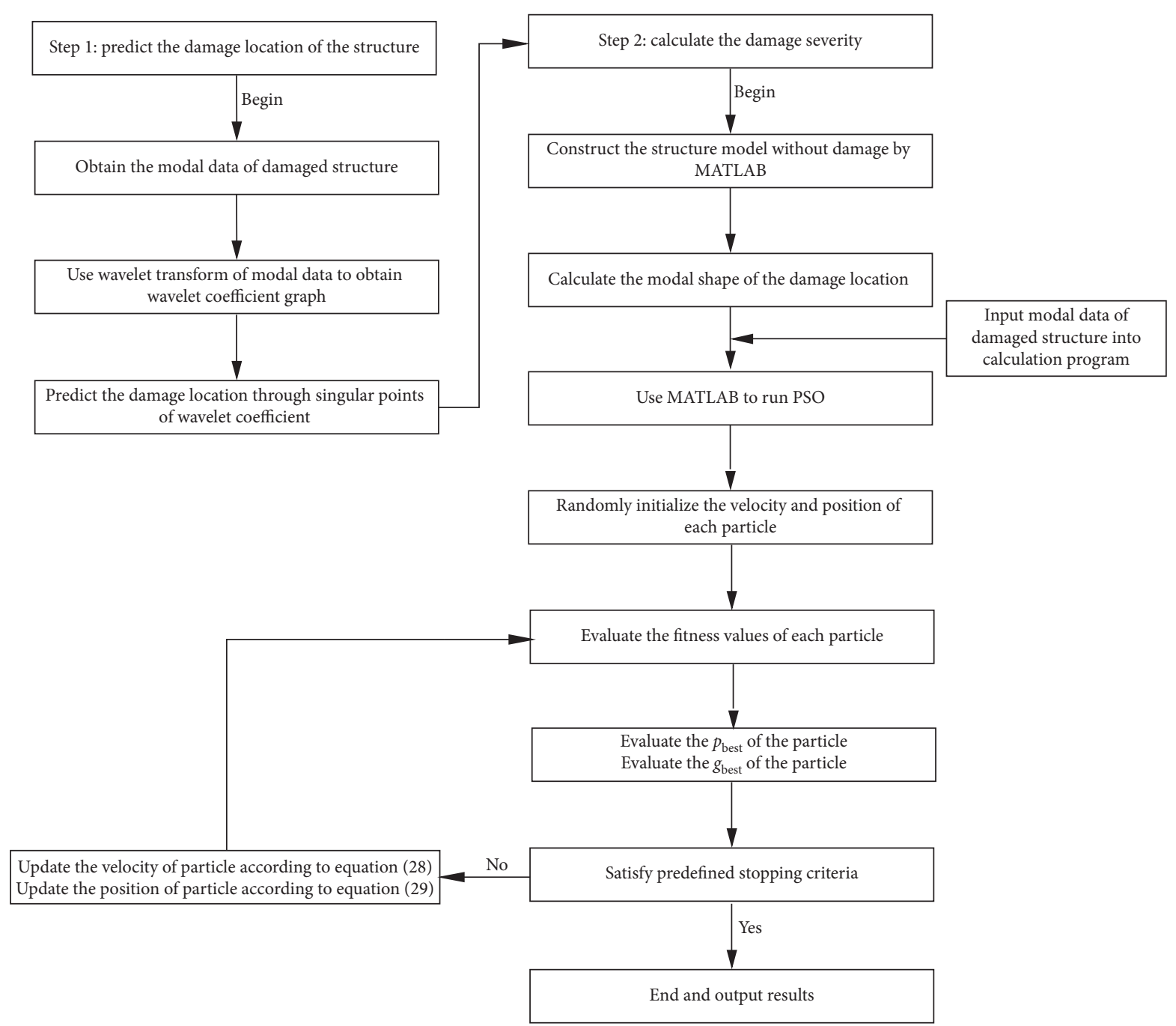

Figure 7: The damage identification flow chart of WPSO.

Taking WPSO as an example, Figure 7 shows the flow chart of structural damage identification combined with wavelet transform and PSO.

\section{Numerical Simulation}

To validate the correctness of the proposed method in nonuniform damage identification and the ability of combining wavelet with IA to identify nonuniform microdamage, the numerical simulation is carried out for the fixed support steel beam. In this study, two scenarios are considered, as shown in Table 3. The modeling parameters of the fixed support steel beam are shown in Table 4. Considering that the size of the vibration sensor used in the experiment is $26 \mathrm{~mm}$, the beam element size is set to $26 \mathrm{~mm}$.

There are two purposes to set scenario 1: (1) to verify that the wavelet-intelligent algorithm can quickly and accurately identify the structural damage; (2) scenario 1 is used as a reference for scenario 2 .
4.1. Selection of Mother Wavelet. MATLAB 2015b is used for wavelet transform. In order to obtain the best wavelet coefficient graph, the suitable mother wavelet has to be selected. Research studies have shown that researchers choose the appropriate mother wavelet based on different criteria. For example, Ovanesova and Suarez [48] selected the mother wavelet based on regularity, symmetry, and the ability to accurately reconstruct the analysis signal. Zhong and Oyadiji [49] selected the mother wavelet based on the number of vanishing moment and the effective support. However, in most cases, there are no fixed rules for the selection of mother wavelet, and the appropriate mother wavelet is based on different situations [50]. In most cases, the trial and error method is applied to select a mother wavelet [51]. In this study, the trial and error method is used to transform the data for many times. According to the results of wavelet transform, DB wavelet is selected for wavelet transform because it can provide the best identification result and resolution for wavelet coefficients. 
TABLE 3: Damaged scenarios of the fixed beam.

\begin{tabular}{|c|c|c|c|c|c|c|c|}
\hline Scenarios & Damage form & $\begin{array}{c}\text { Element } \\
\text { quantities }\end{array}$ & $\begin{array}{l}\text { Element size } \\
(\mathrm{mm})\end{array}$ & $\begin{array}{l}\text { Length of beam } \\
\qquad(\mathrm{mm})\end{array}$ & $\begin{array}{c}\text { Damaged } \\
\text { element }\end{array}$ & $\begin{array}{l}\text { Severity } \\
(\%)\end{array}$ & $\begin{array}{c}\text { Damage depth: } h \text { or } a \\
(\mathrm{~mm})\end{array}$ \\
\hline \multirow{3}{*}{1} & \multirow{3}{*}{ Transfixion crack } & \multirow{3}{*}{43} & \multirow{3}{*}{26} & \multirow{3}{*}{1118} & 12 & 4 & 1.1 \\
\hline & & & & & 25 & 20 & 5.7 \\
\hline & & & & & 38 & 10 & 2.8 \\
\hline \multirow{2}{*}{2} & Semielliptical & \multirow{2}{*}{48} & \multirow{2}{*}{26} & \multirow{2}{*}{1248} & 25 & 7.26 & 7.5 \\
\hline & crack & & & & 38 & 4.87 & 6.2 \\
\hline
\end{tabular}

Table 4: Parameters of the fixed beam.

\begin{tabular}{lc}
\hline Parameters & Value \\
\hline Density $(\rho)$ & $7800 \mathrm{~kg} / \mathrm{m}^{3}$ \\
Poisson's ratio $(v)$ & 0.3 \\
Elasticity modulus $(E)$ & $2.1 \mathrm{GPa}$ \\
Length $(L)$ & $1.3 \mathrm{~m}$ \\
\hline
\end{tabular}

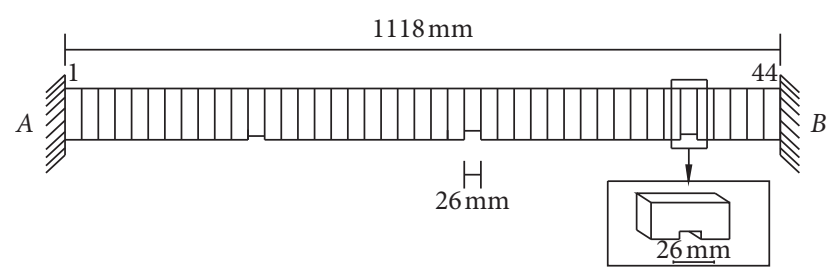

Figure 8: Model of the fixed beam with transfixion crack.

4.2. Scenario 1. Taking a fixed steel beam as the object, the damage form is set as a transfixion crack along the thickness direction, and its model is shown in Figure 8. According to the experimental model in later chapters, the two ends of the fixed beam are rigidly connected. The steel beam is made of Q235 steel, and the section size is $b \times h=60 \mathrm{~mm} \times 80 \mathrm{~mm}$, and the material parameters are shown in Table 4 . According to Table 4, the finite element modeling of Figure 8 is carried out by using ANSYS finite element software. Points $A$ and $B$ of the beam end adopt the fixed support so that the beam end has no displacement in any direction. The finite element model of the fixed beam is divided into 43 elements in the order of $A-B$, as shown in Figure 8, and the element nodes are coded in turn. The damaged elements and damage severity of the fixed beam are set as follows: the damage severity of element 12 is $4 \%$, the damage severity of element 25 is $20 \%$, and the damage severity of element 38 is $10 \%$. The element stiffness is reduced to simulate the damage. The WPSO and WGA are used to identify the damage location and severity of the fixed beam.

4.2.1. Structural Damage Location Identification. The finite element model of the fixed beam is established according to Figure 8 , and the structural displacement mode is calculated by using ANSYS. MATLAB is used to take the second derivative of the structural displacement mode to obtain the strain mode. Then, the wavelet toolbox in MATLAB is used to select the DB wavelet to transform the strain modal data, and the wavelet coefficient is obtained as shown in Figure 9.

Figure 9 depicts that there are sudden changes near points 10, 23, and 36 in the wavelet coefficient graph. The strain mode data, calculated by the second-order derivation of the displacement mode with MATLAB, will be reduced by two. Therefore, points 10,23 , and 36 in the wavelet coefficient diagram actually correspond to elements 12,25 , and 38 in the fixed beam model. Around the damage location, there will be sudden changes that are influenced by the damage, but the maximum value point of the modulus corresponds to the damage location. Thus, it can be judged that the damage of the fixed beam occurs in elements 12,25 , and 38, which is consistent with the damage element setting of the finite element model of the fixed beam and verifies that the wavelet singularity principle can accurately identify the structural transfixion damage location.

4.2.2. Structural Damage Severity Identification. According to Figure 8 , the fixed beam is modeled by using MATLAB. The elements' nodes are numbered in the order of $A-B$, and the element stiffness is assembled according to the numbering order to obtain the overall stiffness, thus completing the modeling. MATLAB is applied to program PSO and GA, respectively, and the damage severity of damaged element identified by wavelet transform can be regarded as the unknown value of fitness function. PSO and GA will run on the same computer ten times to reduce the randomness of the calculation. Due to the randomness of the results calculated by PSO and GA each time, the maximum value and minimum value will not be deleted from the results to ensure the fairness of each calculation result.

(1) As shown in Figures 10 and 11, the structural damage severity and calculation error identified by PSO are shown, respectively.

Figures 10 and 11 show that the accuracy of PSO in structural damage severity identification is high, and the error is within $0.5 \%$. Moreover, the higher the damage severity is, the smaller the damage error fluctuation is. The results also show that PSO has no prematurity and local optimum in the ten calculations, which indicates that PSO can effectively identify the damage severity of the structure with transfixion crack.

(2) As shown in Figures 12 and 13, the structural damage severity and calculation error identified by GA are shown, respectively.

Figures 12 and 13 show that the accuracy of GA in structural damage severity identification is slightly lower than that of PSO, and the error is within $0.55 \%$. Moreover, the higher the damage severity is, the 


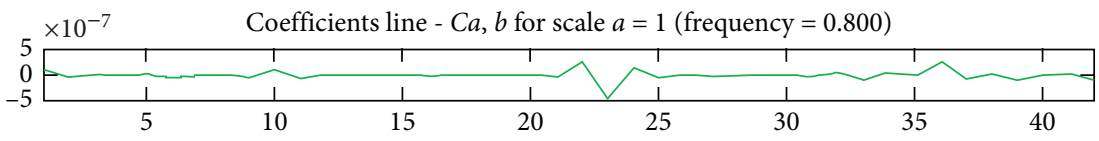

FIgURE 9: Wavelet coefficients.

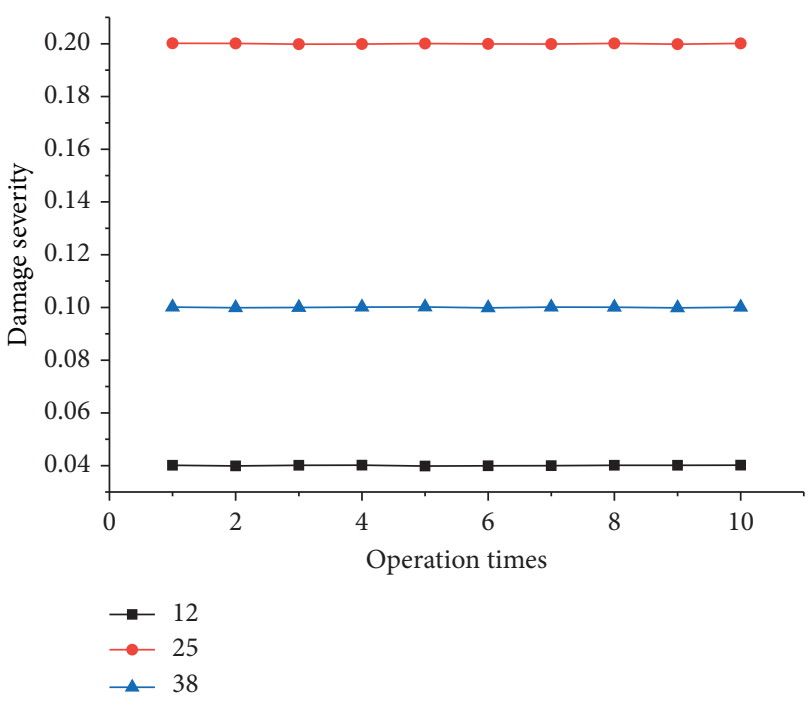

FIgURE 10: Damage severity identification of PSO.

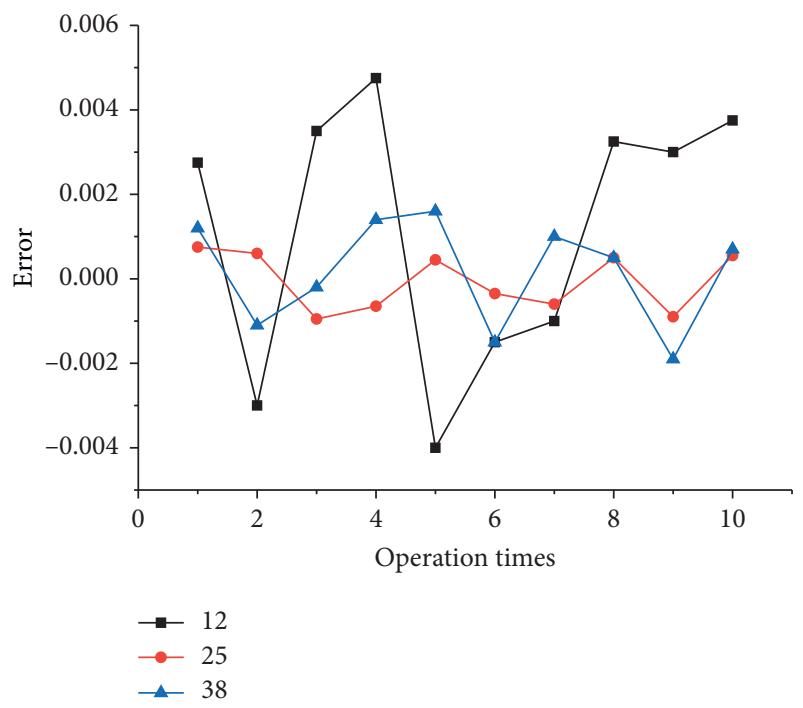

FIgURE 11: Error of PSO.

smaller the damage error fluctuation is. The results also show that GA has no prematurity and local optimum in the ten calculations, which indicates that GA can effectively identify the damage severity of the structure with transfixion crack.

$\mathrm{PSO}^{\prime}$ is defined as the average time of PSO running ten times. Figure 14 depicts the running time ratio of GA, PSO to $\mathrm{PSO}^{\prime}$, and it shows that PSO takes only $1 / 3$ of GA time to identify the damage severity. Figures 11 and 13 show that the identification accuracy of GA and PSO is basically the same.

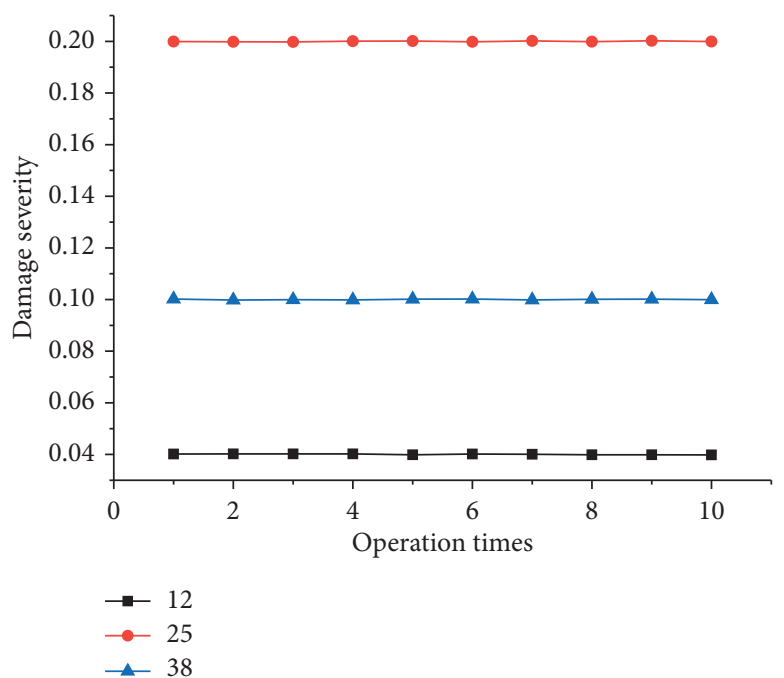

Figure 12: Damage severity identification of GA.

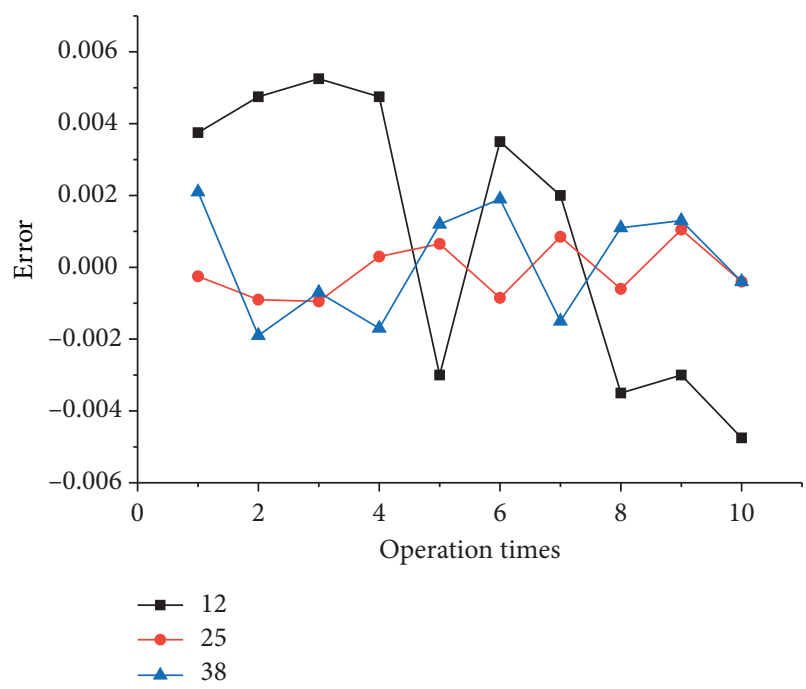

FiguRE 13: Error of GA.

4.3. Scenario 2. Taking a fixed steel beam structure as the object, the damage shape is set as a semielliptical crack in the surface of the fixed beam, and its model is shown in Figure 15. According to the experimental model in later chapters, the two ends of the fixed beam are rigidly connected. The steel beam is made of Q235 steel, and the section size is $b \times h=60 \mathrm{~mm} \times 80 \mathrm{~mm}$, and the material parameters are shown in Table 4. According to Table 4, the finite element modeling of Figure 15 is carried out by using ANSYS finite element software. Points A and B of the beam end adopt the fixed support so that the beam end has no displacement in any direction. The finite element model of the fixed beam is divided 


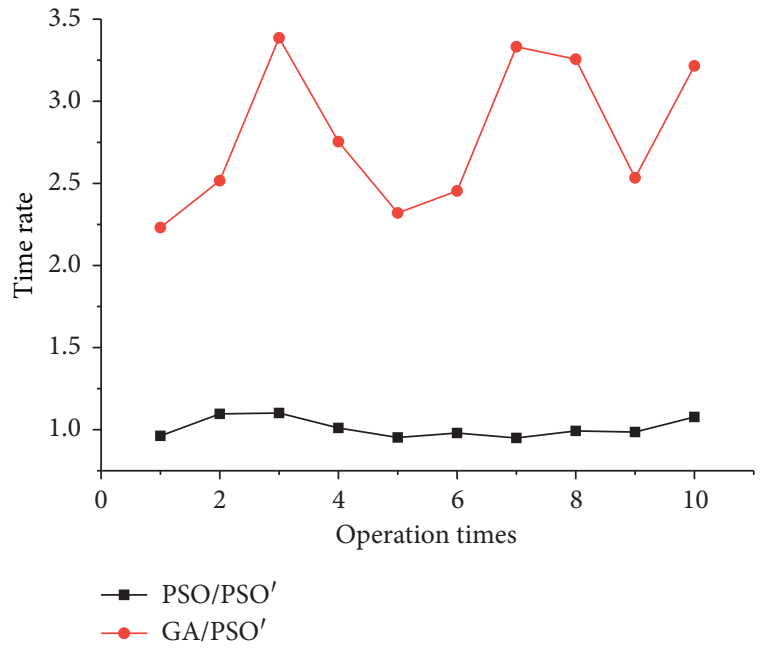

FIgUre 14: Ratio of operation time of PSO and GA to PSO'.

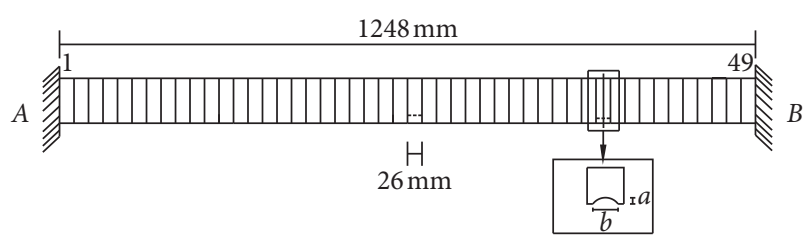

Figure 15: Model of the fixed beam with semielliptical crack.

into 48 elements in the order of $A-B$, as shown in Figure 15, and the element nodes are coded in turn. The damaged elements and damage severity of the fixed beam are set as follows: the damage severity of element 25 is $7.26 \%$ and the damage severity of element 38 is $4.87 \%$, reducing the element stiffness to simulate the damage. The WPSO and WGA are used to identify the damage location and severity of the fixed beam.

4.3.1. Structural Damage Location Identification. The finite element model of the fixed beam is established according to Figure 15, and the structural displacement mode is calculated by using ANSYS. MATLAB is used to take the second derivative of the structural displacement mode to obtain the strain mode. Then, the wavelet toolbox in MATLAB is used to select the DB wavelet to transform the strain modal data, and the wavelet coefficient is obtained as shown in Figure 16.

Figure 16 depicts that there are sudden changes near points 23 and 36 in the wavelet coefficient graph, and the mutation values of points 23 and 36 relative to the surrounding points are the largest. According to the analysis in Section 4.2.1, it can be judged that the damage occurred in elements 25 and 38 of the fixed beam which is consistent with the damage element setting of the finite element model of the fixed beam and verifies that the wavelet singularity principle can accurately identify the structural semielliptical damage location.

4.3.2. Structural Damage Severity Identification. According to Figure 15, the fixed beam is modeled by using MATLAB. The elements' nodes are numbered in the order of $A-B$, and the element stiffness is assembled according to the numbering order to obtain the overall stiffness, thus completing the modeling. MATLAB is applied to program PSO and GA, respectively, and the damage severity of damaged element identified by wavelet transform can be regarded as the unknown value of fitness function. PSO and GA will run on the same computer ten times to reduce the randomness of their calculation. Due to the randomness of the results calculated by PSO and GA each time, the maximum and minimum will not be deleted from the results to ensure the fairness of each calculation result.

(1) Figures 17 and 18 depict the structural damage severity and calculation error identified by PSO, respectively.

Figures 17 and 18 show that the accuracy of PSO in structural damage severity identification is high, and the error is within $0.55 \%$. Moreover, the higher the damage severity is, the smaller the damage error fluctuation is. The results also show that PSO has no prematurity and local optimum in the ten calculations, which indicates that PSO can effectively identify the damage severity of the structure with semielliptical crack.

(2) Figures 19 and 20 depict the structural damage severity and calculation error identified by GA, respectively.

Figures 19 and 20 show that the accuracy of PSO in structural damage severity identification is high, and the error is within $0.55 \%$. Moreover, the higher the damage severity is, the smaller the damage error fluctuation is. The results also show that GA has no prematurity and local optimum in the ten calculations, which indicates that GA can effectively identify the damage severity of the structure with semielliptical crack.

Figure 21 depicts the running time ratio of GA and PSO to $\mathrm{PSO}^{\prime}$, and it shows that PSO takes only $1 / 3$ of GA time to identify the damage severity. Figures 18 and 20 show that the recognition accuracy of GA and PSO is basically the same.

The analyses of Sections 4.2.2 and 4.3.2 show that the accuracy of damage identification of PSO and GA adopted in this study is basically the same, but the time spent by PSO is far less than that of GA, which indicates that PSO is more suitable for damage identification in this study.

According to the damage severity identified by PSO and GA, the semiminor axis $a$ of semielliptical crack is inversely calculated by equation (9), and the calculation results are shown in Table 5. The identification error of the semiminor axis is calculated according to Table 5, as shown in Figures 22(a) and 22(b), which show that the maximum error of semiminor axis $a$ is not more than $0.1 \%$ for element 25 with larger damage severity, while the maximum error of semiminor axis $a$ is not more than $0.15 \%$ for element 38 with smaller damage severity. The maximum errors of damage severity identified by PSO and GA are less than $0.55 \%$. The above shows that the calculation method of the semiminor axis $a$ of the semielliptical crack obtained in this study is very 


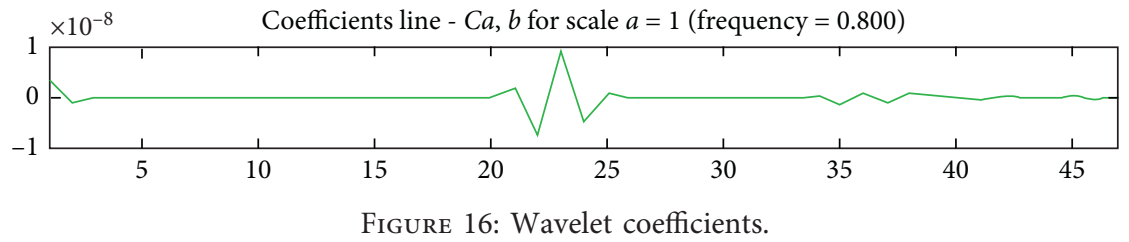

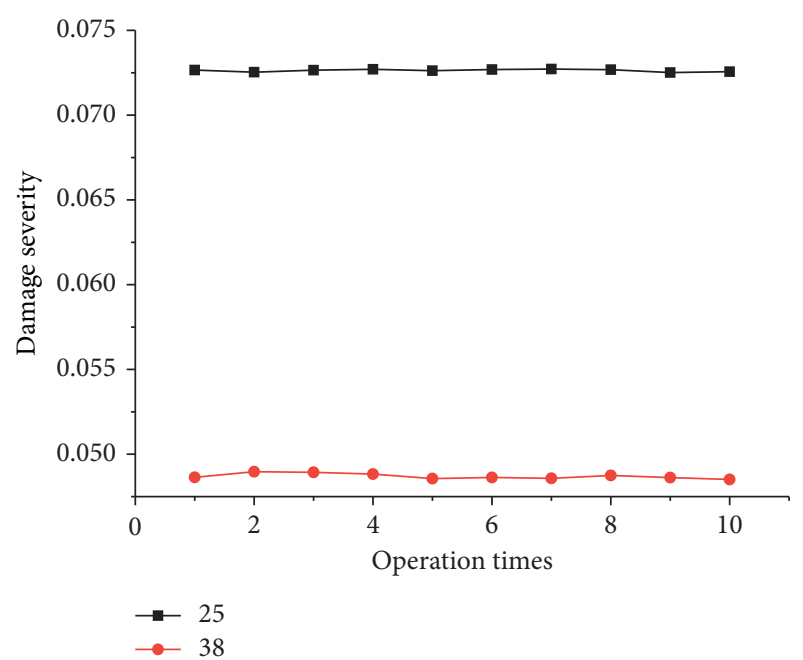

FIgURE 17: Damage severity identification of PSO.

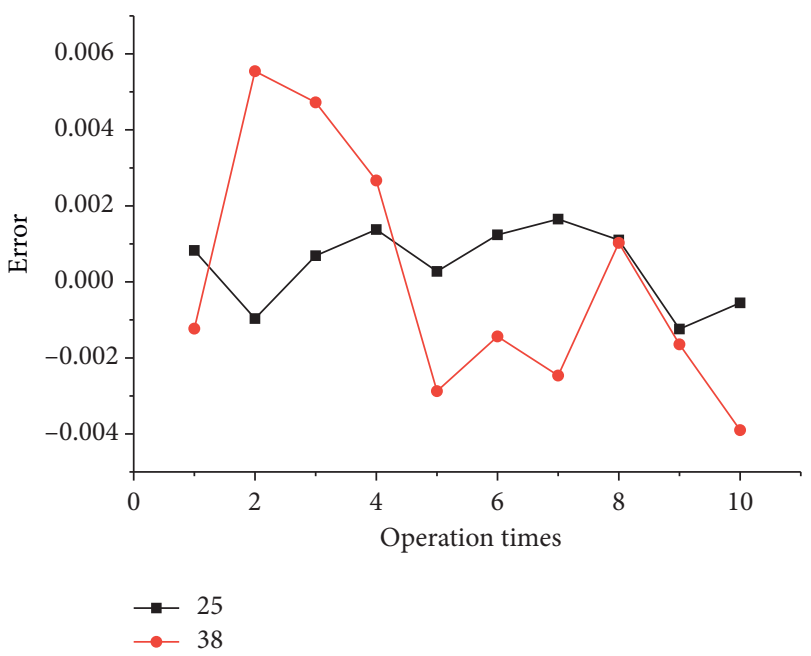

Figure 18: Error of PSO.

accurate, and the error obtained by describing the damage with the semiminor axis is less than that of the damage severity describe the damage.

4.4. Noise Effect on Damage Identification. Noise is inevitable in the real life. To simulate the influence of noise on wavelet transform, we add white noise to the signal calculated by using ANSYS in scenario 2. The signal is transformed by wavelet, and the antinoise ability of wavelet is judged by observing the wavelet coefficient graph. Noise intensity is defined by signal-to-noise ratio (SNR) as follows:

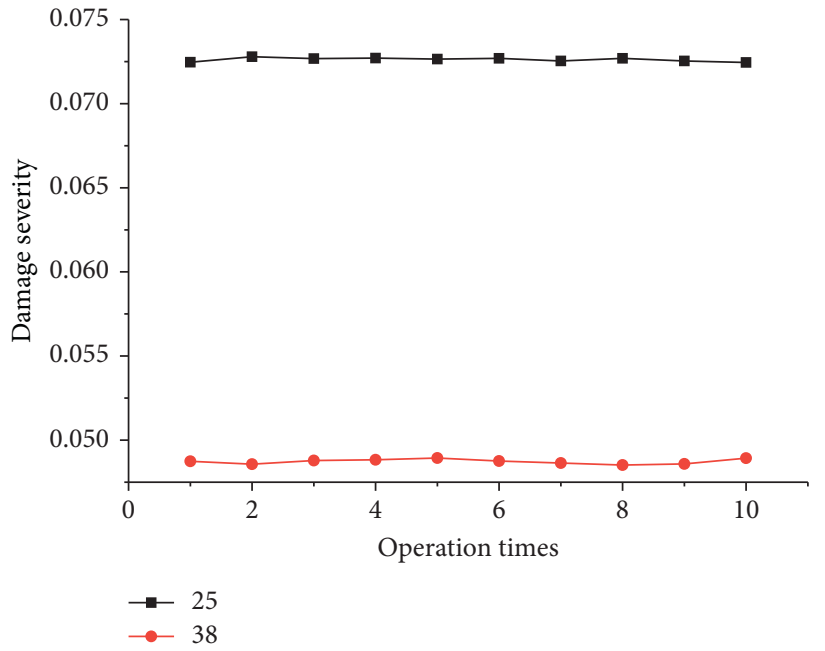

Figure 19: Damage severity identification of PSO.

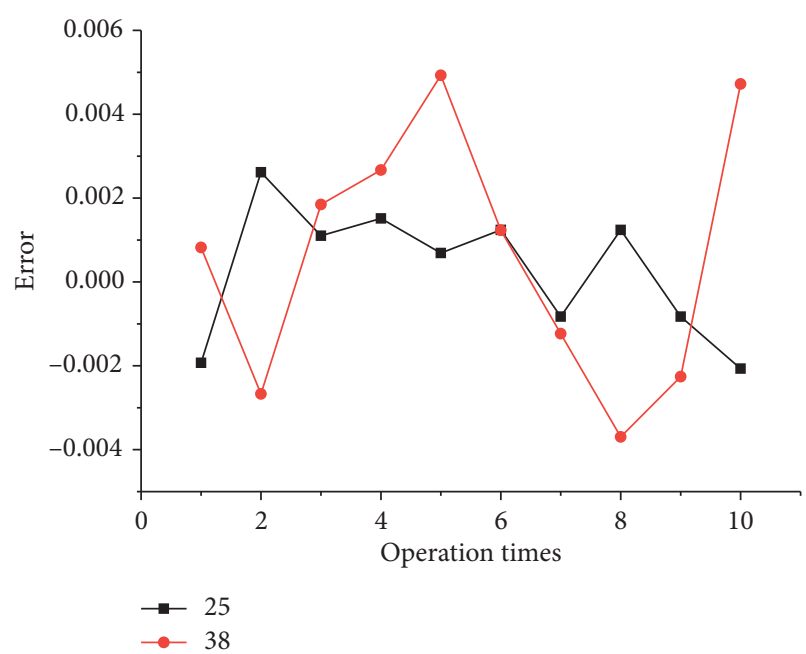

FIgURe 20: Error of PSO.

$$
\operatorname{SNR}(\mathrm{d} B)=20 \log _{10} \frac{A_{S}}{A_{N}},
$$

where $A_{S}$ and $A_{N}$ are the root-mean-square value of displacement mode signal and noise, respectively.

The SNRs 10 and $15 \mathrm{~dB}$ are applied to study the influence of different noise levels on damage identification. Figure 23 depicts the wavelet coefficient of numerical simulation of the fixed beam with semielliptical crack. Wavelet transform is applied to the numerical simulation data added with noise, and the wavelet coefficient graphs of noise influence are shown in Figure 24. It can be seen from Figure 24 that the 


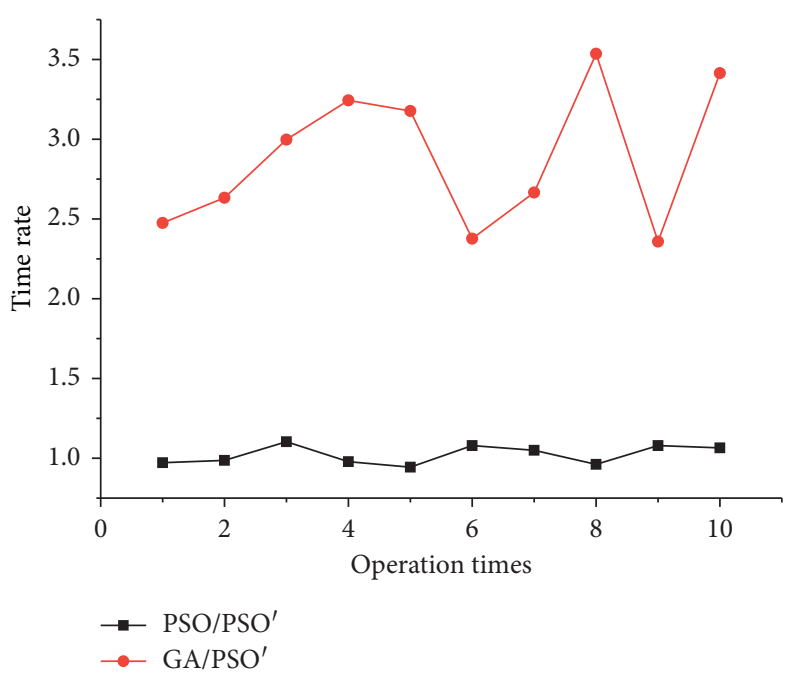

Figure 21: Ratio of operation time of PSO and GA to PSO'.

TABLE 5: Calculation value of $a$.

\begin{tabular}{lcccc}
\hline No. & \multicolumn{2}{c}{$\begin{array}{c}\text { Element 25, } a \\
(\mathrm{~mm})\end{array}$} & \multicolumn{2}{c}{$\begin{array}{c}\text { Element 38, } a \\
(\mathrm{~mm})\end{array}$} \\
& PSO & GA & PSO & GA \\
\hline 1 & 7.5042 & 7.4942 & 6.1949 & 6.2009 \\
2 & 7.4977 & 7.5106 & 6.2149 & 6.1906 \\
3 & 7.5037 & 7.5052 & 6.2125 & 6.204 \\
4 & 7.5062 & 7.5067 & 6.2064 & 6.2064 \\
5 & 7.5022 & 7.5037 & 6.19 & 6.2131 \\
6 & 7.5057 & 7.5057 & 6.1942 & 6.2022 \\
7 & 7.5072 & 7.4982 & 6.1912 & 6.1949 \\
8 & 7.5052 & 7.5057 & 6.2015 & 6.1876 \\
9 & 7.4967 & 7.4982 & 6.1936 & 6.1918 \\
10 & 7.4992 & 7.4982 & 6.1869 & 6.2125 \\
Actual value, $a(\mathrm{~mm})$ & \multicolumn{3}{c}{7.5} & \multicolumn{3}{c}{6.2} \\
\hline
\end{tabular}

noise has no obvious influence on the damage location by wavelet analysis, and the damage location can be clearly located through the wavelet coefficient. This is because the displacement mode is less sensitive to noise, and the influence of noise will be further reduced after the displacement mode is calculated to obtain the strain mode. The wavelet coefficients of the signal are obtained after wavelet transform, which also reduces the influence of noise on the signal. Therefore, it can be inferred that under the influence of noise, wavelet transform can work well in semielliptical crack identification.

\section{Experimental Study}

5.1. Experimental Setup and Procedure. To study the effectiveness of the proposed method in calculating the nonuniform damage, an experimental investigation is carried out on the fixed beam, as shown in Figure 25. The transfixion crack and semielliptical crack are shown in Figure 26, which are manufactured by a milling machine, and the accuracy of manufactured damage is $0.1 \mathrm{~mm}$. The Q235 rectangular steel beam with a span of $1300 \mathrm{~mm}$ is adopted, and the section size is $b \times h=60 \mathrm{~mm} \times 80 \mathrm{~mm}$. The beam's material properties are as follows. Density is $\rho=7800 \mathrm{~kg} / \mathrm{m}^{3}$, Poisson's ratio is $\mu=0.3$, and elastic modulus is $E=2.1 \times 10^{11} \mathrm{~N} / \mathrm{m}^{2}$. The two ends of the beam are fixed as shown in Figure 25. The detailed experimental scenarios of the beam are shown in Table 6, and the stiffness reduction method is used to simulate the damage severity.

Taking scenario 2 as an example, the excitation method, the element division of the beam, and the arrangement of the sensor are introduced. The experimental devices and sensor layout are shown in Figure 25. The beam is excited by using the hammer, and the excitation points are selected randomly on the upper surface of the beam. In Figure 25(a), the node division method of the beam is as follows. The middle point of the beam is taken as the symmetrical point to divide the element at both ends. After the element is divided, the end node of the first element at one end is taken as node 1 to the last element at the other end for node numbering in turn. Sixteen high sensitivity vibration sensors are used to collect the vibration data of the beam structural unit nodes. Node 1 is taken as the reference point, and the sensors are arranged on the central axis of the upper surface of the beam according to the node number successively from node 1 , as shown in Figures 25(a) and 25(b). According to the quantity of nodes to be measured, the vibration data of all nodes can be collected in scenario 2 after 4 times of experiments. The node numbering rule and sensor arrangement rule of scenario 1 are the same as that of scenario 2. The collected vibration data are processed with a DH5922D analysis system to obtain the first three orders of displacement modal data of the beam structure. Then, the corresponding strain modal data can be calculated by taking the second derivative of the displacement modal data in MATLAB. The wavelet coefficients of the first three strain modes of the beam structure in scenario 2 are shown in Figure 27. The first three orders of the strain mode are calculated with the wavelet transform, and it is determined that the second-order strain mode has the best identification effect. 


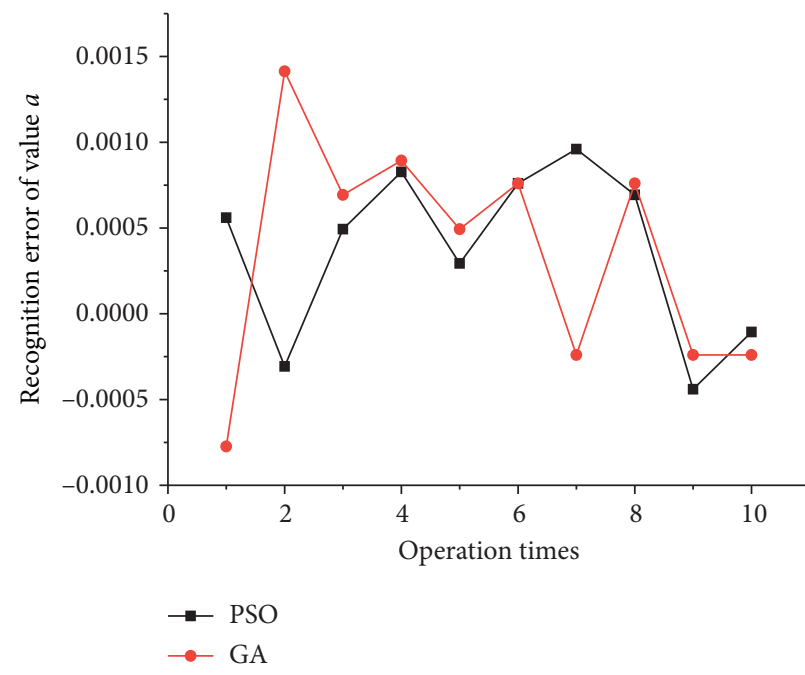

(a)

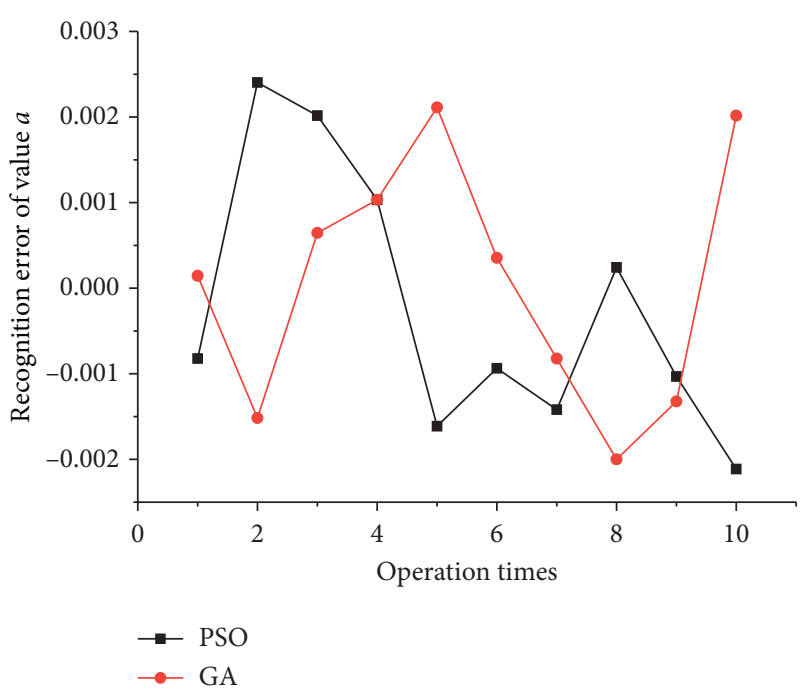

(b)

FIGURE 22: Identification error of semiminor axis: (a) identification error of element 25; (b) identification error of element 38.

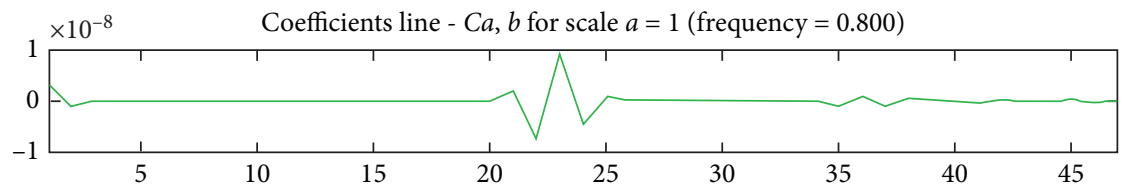

Figure 23: Wavelet coefficient of beams with semielliptical cracks without noise influence.

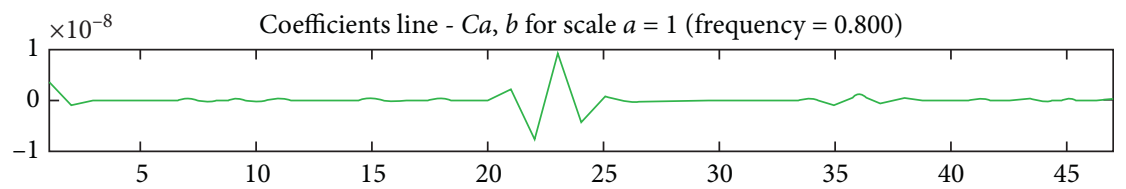

(a)

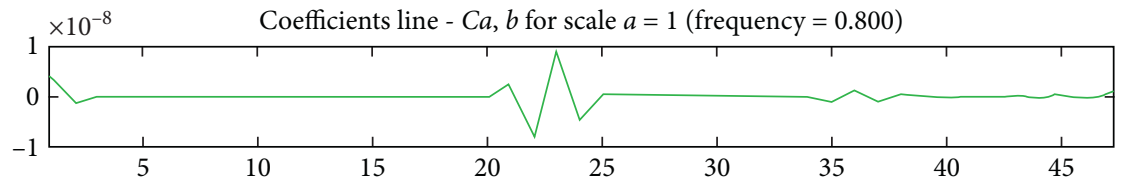

(b)

FIGURE 24: Wavelet coefficient of beams with semielliptical cracks influenced by noise: (a) SNR $=10 \mathrm{~dB}$; (b) SNR $=15 \mathrm{~dB}$.

5.2. Experimental Results. Figure 28 shows the wavelet coefficient graph of the beam structural second-order strain modal data after being denoised and wavelet transformed. Because the MATLAB wavelet toolbox cannot denoise an odd number of data points, the datum of node 49 in scenario 2 is deleted. As can be seen in Figure 28, because of noise in the experimental data, the damaged and nondamaged points have singular values after denoising by the wavelet toolbox. The structural damage location corresponds to the modulus maximum point in the numerical simulation, and the modulus maximum point in the range of singular points in Figure 28 is the damaged point, which corresponds to the damage location of the beam. As shown in Figure 28(a), the damage information that corresponds to element 12 in scenario 1 has been covered by noise, which indicates that the damage information is easily affected by noise and other environmental factors in the structural microdamage identification experiment. Figure 28(b) depicts the wavelet coefficient graph of scenario 2, which shows that the wavelet coefficient points that correspond to the damage element have mutations. The above analysis shows that the minimum damage severity that can be identified by the structural damage experiment is between $4 \%$ and $5 \%$ due to the interference of noise. PSO and GA are used to calculate the 


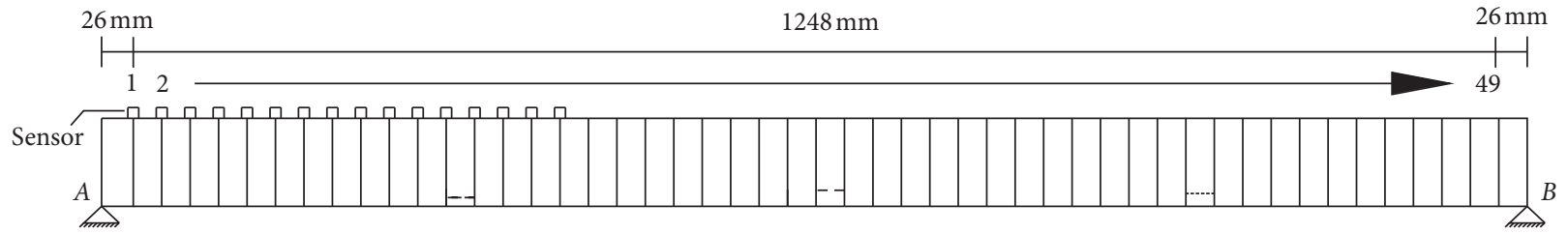

(a)

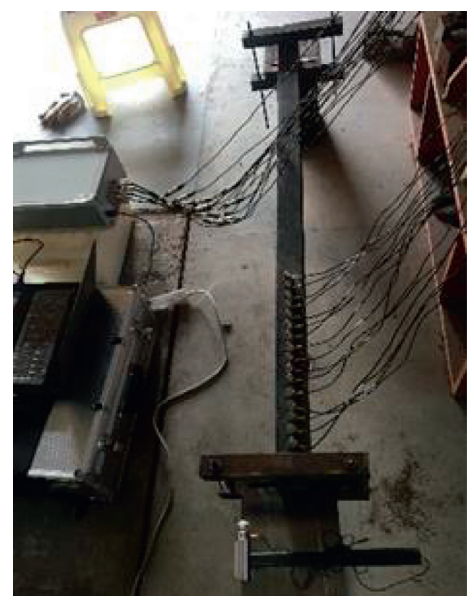

(b)

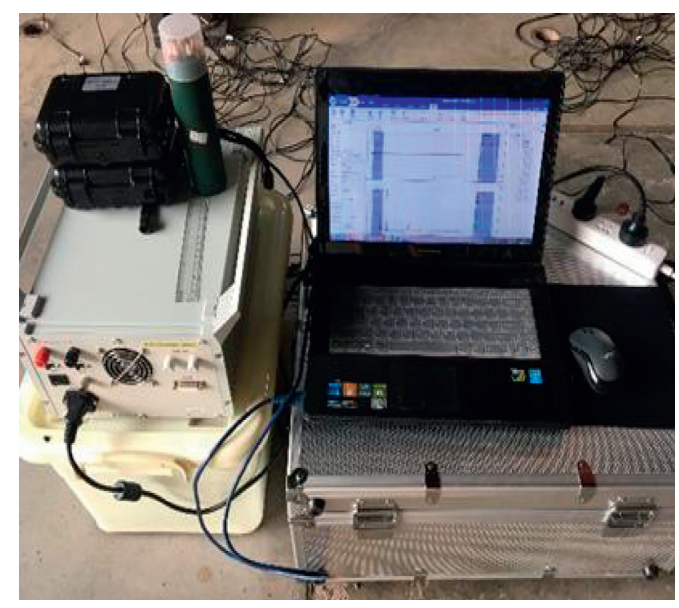

(c)

Figure 25: Experimental device layout: (a) sensor layout; (b) experimental beam; (c) experimental equipment.

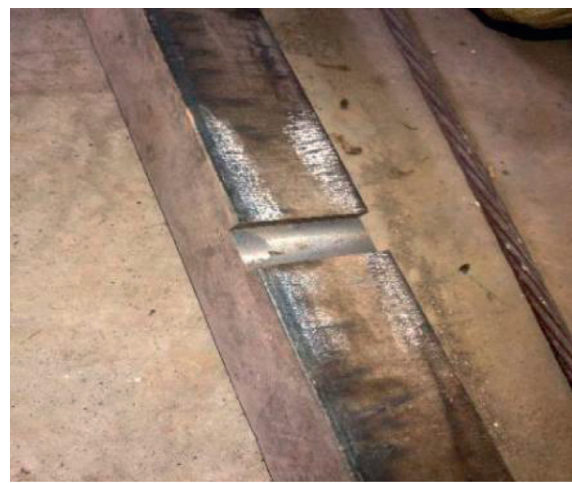

(a)

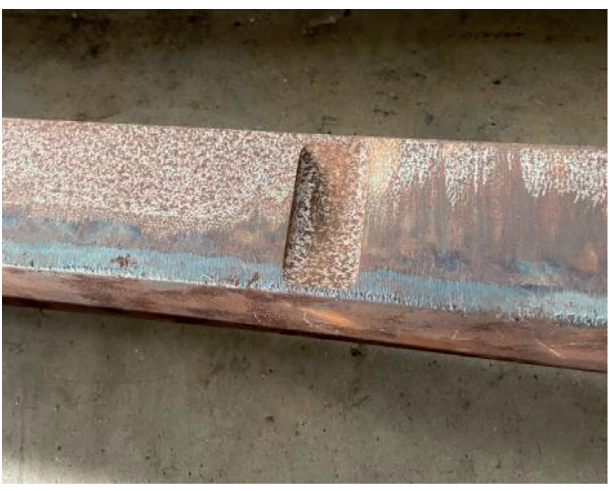

(b)

FiguRE 26: Damage form: (a) transfixion crack; (b) semielliptical crack. 
Table 6: Details of experiment scenarios.

\begin{tabular}{|c|c|c|c|c|c|c|c|}
\hline No. & $\begin{array}{l}\text { Element } \\
\text { number }\end{array}$ & $\begin{array}{c}\text { Element size } \\
(\mathrm{mm})\end{array}$ & $\begin{array}{l}\text { Length } \\
(\mathrm{mm})\end{array}$ & $\begin{array}{c}\text { Damaged } \\
\text { element }\end{array}$ & Form & $\begin{array}{c}\text { Severity } \gamma \\
(\%)\end{array}$ & Damage depth $h$ or $a(\mathrm{~mm})$ \\
\hline \multirow{3}{*}{1} & \multirow{3}{*}{43} & \multirow{3}{*}{26} & \multirow{3}{*}{1118} & 12 & \multirow{3}{*}{ Transfixion } & 4 & 1.1 \\
\hline & & & & 25 & & 20 & 5.7 \\
\hline & & & & 38 & & 10 & 2.8 \\
\hline \multirow{2}{*}{2} & \multirow{2}{*}{48} & \multirow{2}{*}{26} & \multirow{2}{*}{1248} & 25 & \multirow{2}{*}{ Semiellipse } & 7.26 & 7.5 \\
\hline & & & & 38 & & 4.87 & 6.2 \\
\hline
\end{tabular}

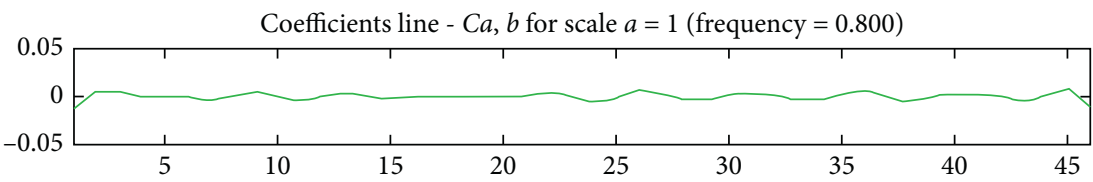

(a)

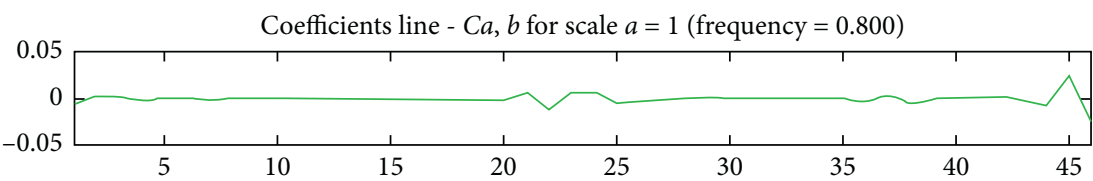

(b)

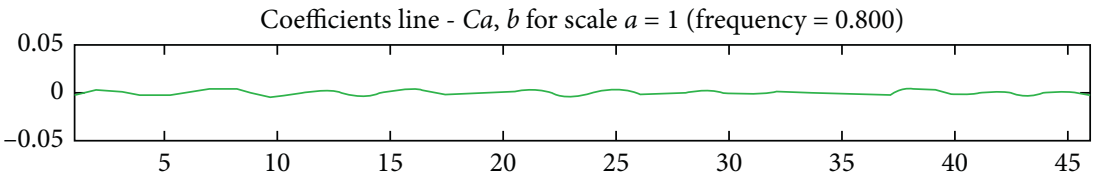

(c)

FIGURE 27: Wavelet coefficients of the strain mode in scenario 2: wavelet coefficient of (a) the first-order strain mode; (b) the second-order strain mode; (c) the third-order strain mode.

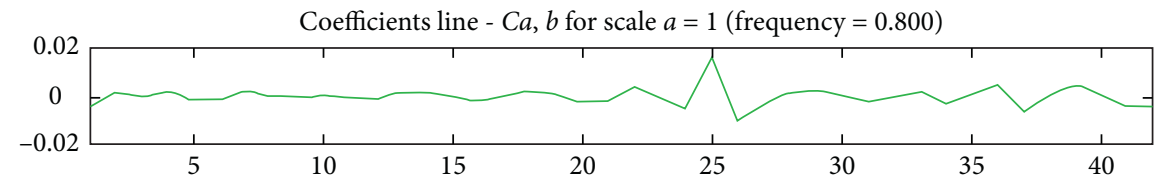

(a)

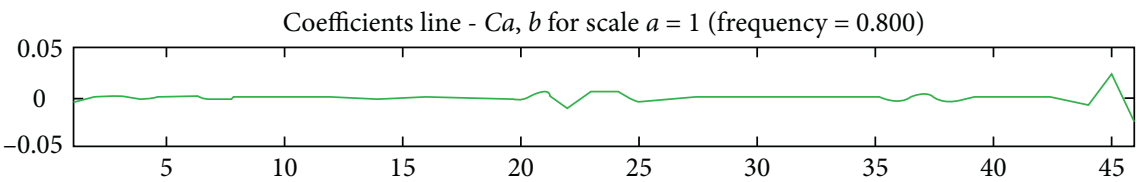

(b)

Figure 28: Wavelet coefficients of the second-order strain mode of the experimental beam: (a) scenario 1; (b) scenario 2.

damage severity of the beam under 2 scenarios with 10 runs each, and the calculation results are shown in Table 7 . The semiminor axis $a^{\prime}$ of the semielliptical crack can be inversely calculated by equation (9) and Table 7 , and the results are shown in Tables 8 and 9. As show in Tables 7-9, the minimum absolute error of PSO in identifying the damage severity of scenario 2 is $1.1184 \%$, with a corresponding $a^{\prime}$ of $6.8409 \mathrm{~mm}$ and an error of $0.6409 \mathrm{~mm}$; the minimum absolute error of GA in identifying the damage severity of scenario 2 is $1.3993 \%$, with a corresponding $a^{\prime}$ of $6.9922 \mathrm{~mm}$ and an error of $0.7922 \mathrm{~mm}$.
As demonstrated by the above analyses, WPSO and WGA can effectively identify the damage location within the beam and the severity of the damage in both theory and experiments. Furthermore, the semielliptical crack calculation formula can be used to accurately calculate the structural damage size. Multiple factors in the experiment influence its accuracy, including environmental noise, and errors in the manufacturing process. These factors can reduce the efficacy of the damage severity identification by PSO and GA and introduce errors in the calculation results of semielliptical damage. However, the calculation results 
TABLE 7: Calculation of damage severity $\gamma^{\prime}$.

\begin{tabular}{|c|c|c|c|c|}
\hline \multirow{2}{*}{ No. } & \multicolumn{2}{|c|}{$\gamma^{\prime}$ of element 25} & \multicolumn{2}{|c|}{$\gamma^{\prime}$ of element 38} \\
\hline & PSO & GA & $\mathrm{PSO}$ & GA \\
\hline 1 & 0.084101 & 0.084354 & 0.060135 & 0.062913 \\
\hline 2 & 0.084512 & 0.084139 & 0.059943 & 0.062859 \\
\hline 3 & 0.084435 & 0.084656 & 0.059884 & 0.063121 \\
\hline 4 & 0.084463 & 0.084465 & 0.059895 & 0.062798 \\
\hline 5 & 0.084224 & 0.084792 & 0.059892 & 0.062957 \\
\hline 6 & 0.084343 & 0.084774 & 0.060012 & 0.062693 \\
\hline 7 & 0.084369 & 0.084258 & 0.060149 & 0.062974 \\
\hline 8 & 0.084162 & 0.084396 & 0.060098 & 0.062865 \\
\hline 9 & 0.084143 & 0.084552 & 0.060113 & 0.062937 \\
\hline 10 & 0.084295 & 0.083801 & 0.060154 & 0.062716 \\
\hline$\gamma$ & \multicolumn{2}{|c|}{0.0726} & \multicolumn{2}{|c|}{0.0487} \\
\hline
\end{tabular}

TABLE 8: Calculation results of $a^{\prime}$ based on PSO.

\begin{tabular}{lcccc}
\hline \multirow{2}{*}{ No. } & \multicolumn{2}{c}{ Element 25} & \multicolumn{2}{c}{ Element 38} \\
& $a^{\prime}(\mathrm{mm})$ & $a-a^{\prime}(\mathrm{mm})$ & $a^{\prime}(\mathrm{mm})$ & $a-a^{\prime}(\mathrm{mm})$ \\
\hline 1 & 8.0548 & 0.5548 & 6.8546 & 0.6546 \\
2 & 8.0741 & 0.5741 & 6.8441 & 0.6441 \\
3 & 8.0705 & 0.5705 & 6.8409 & 0.6409 \\
4 & 8.0718 & 0.5718 & 6.8415 & 0.6415 \\
5 & 8.0606 & 0.5606 & 6.8414 & 0.6414 \\
6 & 8.0662 & 0.5662 & 6.8479 & 0.6479 \\
7 & 8.0674 & 0.5674 & 6.8554 & 0.6554 \\
8 & 8.0577 & 0.5577 & 6.8526 & 0.6526 \\
9 & 8.0568 & 0.5568 & 6.8534 & 0.6534 \\
10 & 8.0639 & 0.5639 & 6.8556 & 0.6556 \\
\hline
\end{tabular}

TABLE 9: Calculation results of $a$ ' based on GA.

\begin{tabular}{lcccc}
\hline \multirow{2}{*}{ No. } & \multicolumn{2}{c}{ Element 25 } & \multicolumn{2}{c}{ Element 38 } \\
& $a^{\prime}(\mathrm{mm})$ & $a-a^{\prime}(\mathrm{mm})$ & $a^{\prime}(\mathrm{mm})$ & $a-a^{\prime}(\mathrm{mm})$ \\
\hline 1 & 8.0667 & 0.5667 & 7.0039 & 0.8039 \\
2 & 8.0566 & 0.5566 & 7.0011 & 0.8011 \\
3 & 8.0808 & 0.5808 & 7.015 & 0.815 \\
4 & 8.0719 & 0.5719 & 6.9978 & 0.7978 \\
5 & 8.0871 & 0.5871 & 7.0063 & 0.8063 \\
6 & 8.0863 & 0.5863 & 6.9922 & 0.7922 \\
7 & 8.0622 & 0.5622 & 7.0072 & 0.8072 \\
8 & 8.0686 & 0.5686 & 7.0014 & 0.8014 \\
9 & 8.0759 & 0.5759 & 7.0052 & 0.8052 \\
10 & 8.0408 & 0.5408 & 6.9934 & 0.7934 \\
\hline
\end{tabular}

demonstrate that the calculation formula for semielliptical damage is an important reference value and has value for engineering applications.

\section{Conclusions}

Due to the complexity of nonuniform crack damage identification, two methods are proposed: (1) a method to identify the structural nonuniform microdamage based on the wavelet-intelligent algorithm and (2) a simplified calculation method for nonuniform crack based on fracture mechanics. By simplifying the nonuniform microcrack into a semielliptical crack, the relationship between the damage severity of the semielliptical crack and the semiminor axis of the crack can be obtained by mathematical derivation and fitting. The validity and accuracy of the crack damage identification method based on the wavelet-intelligent algorithm are verified by notch damage identification. Based on the numerical simulations and experimental study, the main conclusions obtained are as follows:

(1) The wavelet transform of the strain modal can be used to effectively identify the location of structural nonuniform cracks, and this method is robust against noise. However, the smaller damage information will be submerged by larger environmental factors, which makes the structural damage location cannot be accurately identified.

(2) The numerical simulation shows that the wavelet analysis can identify a minimum damage severity of $4 \%$, while in the experiment, the wavelet can identify a minimum range of damage severity from $4 \%$ to $5 \%$ due to interference from external factors. This indicates that the proposed method can identify smaller structural damage when there are fewer external factors.

(3) Although PSO and GA have some errors in identifying the structural damage severity, they can still be used to effectively identify the severity of nonuniform structural damage. The calculation results for the semiminor axis of the semielliptical crack show that the proposed formula can accurately calculate the damage size based on the damage severity. This means that the proposed methods have a significant theoretical foundation and practical engineering value for nonuniform damage identification.

The simplified calculation method for nonuniform cracks and the damage identification method based on the wavelet-intelligent algorithm show great potential in both numerical simulation and experiment. The simulations and experiments of notch damage verified the validity and correctness of the calculation method and identification method of nonuniform microcrack damage in this study. Noise and other influences in the experiment reduce the effectiveness of damage severity identification. Further work needs to be done on reducing these external influences to 
improve the identification efficiency of IA. The work environment of practical engineering applications is complex and changing. To address the challenges that this presents, the problems described above must be solved to allow the structural nonuniform damage identification method (based on the wavelet-intelligent algorithm) and the semielliptical damage calculation method to be reliably applied to realworld structures.

\section{Data Availability}

The data used to support the findings of this study are available from the corresponding author upon request.

\section{Conflicts of Interest}

The authors declare that they have no conflicts of interest regarding the publication of this paper.

\section{Acknowledgments}

This study was financially supported by the National Natural Science Foundation of China (no. 51378079) and the Hunan Graduate Scientific Research Innovation Project of China (no. CX20190636).

\section{References}

[1] J. Xiang and M. Liang, "A two-step approach to multi-damage detection for plate structures," Engineering Fracture Mechanics, vol. 91, pp. 73-86, 2012.

[2] S. A. Ravanfar, H. A. Razak, Z. Ismail, and S. J. S. Hakim, “A two-step damage identification approach for beam structures based on wavelet transform and genetic algorithm," Meccanica, vol. 51, no. 3, pp. 635-653, 2016.

[3] R. Janeliukstis, S. Rucevskis, M. Wesolowski, and A. Chate, "Experimental structural damage localization in beam structure using spatial continuous wavelet transform and mode shape curvature methods," Measurement, vol. 102, pp. 253-270, 2017.

[4] S. Khatir, K. Dekemele, M. Loccufier, T. Khatir, and M. Abdel Wahab, "Crack identification method in beam-like structures using changes in experimentally measured frequencies and Particle Swarm Optimization," Comptes Rendus Mécanique, vol. 346, no. 2, pp. 110-120, 2018.

[5] L. F. Zhu, L. L. Ke, X. Q. Zhu, Y. Xiang, and Y. S. Wang, "Crack identification of functionally graded beams using continuous wavelet transform," Composite Structures, vol. 210, pp. 473-485, 2019.

[6] W. Xu, K. Ding, J. Liu, M. Cao, M. Radzieński, and W. Ostachowicz, "Non-uniform crack identification in platelike structures using wavelet 2D modal curvature under noisy conditions," Mechanical Systems and Signal Processing, vol. 126, pp. 469-489, 2019.

[7] S. W. Doebling, C. R. Farrar, and M. B. Prime, "A summary review of vibration-based damage identification methods," The Shock and Vibration Digest, vol. 30, no. 2, pp. 91-105, 1998.

[8] W. Fan and P. Qiao, "Vibration-based damage identification methods: a review and comparative study," Structural Health Monitoring, vol. 10, no. 1, pp. 83-111, 2011.
[9] E. Peter and P Carden, "Vibration based condition monitoring: a review," Structural Health Monitoring, vol. 3, no. 4, pp. 355-377, 2004.

[10] Y. J. Yan, L. Cheng, Z. Y. Wu, and L. H. Yam, "Development in vibration-based structural damage detection technique," Mechanical Systems and Signal Processing, vol. 21, no. 5, pp. 2198-2211, 2007.

[11] P. Cawley and R. D. Adams, "The location of defects in structures from measurements of natural frequencies," The Journal of Strain Analysis for Engineering Design, vol. 14, no. 2, pp. 49-57, 1979.

[12] G.-R. Gillich and Z.-I. Praisach, "Modal identification and damage detection in beam-like structures using the power spectrum and time-frequency analysis," Signal Processing, vol. 96, pp. 29-44, 2014.

[13] N. T. Khiem and L. K. Toan, "A novel method for crack detection in beam-like structures by measurements of natural frequencies," Journal of Sound and Vibration, vol. 333, no. 18, pp. 4084-4103, 2014.

[14] R. P. Bandara, T. H. T. Chan, and D. P. Thambiratnam, "Frequency response function based damage identification using principal component analysis and pattern recognition technique," Engineering Structures, vol. 66, pp. 116-128, 2014.

[15] I. M. D. Rosa, C. Santulli, and F. Sarasini, "Acoustic emission for monitoring the mechanical behaviour of natural fibre composites: a literature review," Composites Part A Applied science \& Manufacturing, vol. 40, no. 9, pp. 1456-1469, 2009.

[16] W. Fan and P. Qiao, "A 2-D continuous wavelet transform of mode shape data for damage detection of plate structures," International Journal of Solids and Structures, vol. 46, no. 2526, pp. 4379-4395, 2009.

[17] X. Q. Zhu and S. S. Law, "Wavelet-based crack identification of bridge beam from operational deflection time history," International Journal of Solids and Structures, vol. 43, no. 7-8, pp. 2299-2317, 2006.

[18] W.-Y. He, S. Zhu, and W.-X. Ren, "Two-phase damage detection of beam structures under moving load using multiscale wavelet signal processing and wavelet finite element model," Applied Mathematical Modelling, vol. 66, pp. 728744, 2019.

[19] J. Zhou and Z. Li, "Damage detection based on vibration for composite sandwich panels with truss core," Composite Structures, vol. 229, 2019.

[20] H. Hao and Y. Xia, "Vibration-based damage detection of structures by genetic algorithm," Journal of Computing in Civil Engineering, vol. 16, no. 3, pp. 222-229, 2002.

[21] S. Sahu and B. B. Nayak, "An adaptive genetic algorithm method for damage detection in structural elements," Materials Today: Proceedings, vol. 26, 2020.

[22] O. Begambre and J. E. Laier, "A hybrid Particle Swarm Optimization-Simplex algorithm (PSOS) for structural damage identification," Advances in Engineering Software, vol. 40, no. 9, pp. 883-891, 2009.

[23] B. Nanda, D. Maity, and D. K. Maiti, "Vibration based structural damage detection technique using particle swarm optimization with incremental swarm size," International Journal of Aeronautical and Space Sciences, vol. 13, no. 3, pp. 323-331, 2012.

[24] N. B. Guedria, "An accelerated differential evolution algorithm with new operators for multi-damage detection in plate-like structures," Applied Mathematical Modelling, vol. 80, pp. 366-383, 2020.

[25] S. Moradi, P. Razi, and L. Fatahi, "On the application of bees algorithm to the problem of crack detection of beam-type 
structures," Computers and Structures, vol. 89, no. 23-24, pp. 2169-2175, 2011.

[26] H. Y. Guo and Z. L. Li, "Structural damage identification based on Bayesian theory and improved immune genetic algorithm," Expert Systems with Applications, vol. 39, no. 7, pp. 6426-6434, 2012.

[27] L. $\mathrm{Yu}$ and $\mathrm{P} . \mathrm{Xu}$, "Structural health monitoring based on continuous ACO method," Microelectronics Reliability, vol. 51, no. 2, pp. 270-278, 2011.

[28] Y. Zhao, Q. Yan, Z. Yang, X. Yu, and B. Jia, "A novel artificial bee colony algorithm for structural damage detection," Advances in Civil Engineering, vol. 2020, no. 6, 21 pages, Article ID 3743089, 2020.

[29] S. A. Ravanfar, H. Abdul Razak, Z. Ismail, and S. J. S. Hakim, "A hybrid wavelet based-approach and genetic algorithm to detect damage in beam-like structures without baseline data," Experimental Mechanics, vol. 56, no. 8, pp. 1411-1426, 2016.

[30] S. S. Nanthakumar, T. Lahmer, X. Zhuang, G. Zi, and T. Rabczuk, "Detection of material interfaces using a regularized level set method in piezoelectric structures," Inverse Problems in Science and Engineering, vol. 24, no. 1, pp. 153176, 2016.

[31] C. Anitescu, E. Atroshchenko, N. Alajlan, and T. Rabczuk, "Artificial neural network methods for the solution of second order boundary value problems," Computers, Materials \& Continua, vol. 59, no. 1, pp. 345-359, 2019.

[32] R. Perera, S.-E. Fang, and A. Ruiz, "Application of particle swarm optimization and genetic algorithms to multiobjective damage identification inverse problems with modelling errors," Meccanica, vol. 45, no. 5, pp. 723-734, 2010.

[33] S. M. Seyedpoor, "A two stage method for structural damage detection using a modal strain energy based index and particle swarm optimization," International Journal of Non-linear Mechanics, vol. 47, no. 1, pp. 1-8, 2012.

[34] F. Jiang, H. Xia, Q. Anh Tran, Q. Minh Ha, N. Quang Tran, and J. Hu, "A new binary hybrid particle swarm optimization with wavelet mutation," Knowledge-Based Systems, vol. 130, no. 15, pp. 90-101, 2017.

[35] V. Meruane and W. Heylen, "An hybrid real genetic algorithm to detect structural damage using modal properties," $\mathrm{Me}$ chanical Systems and Signal Processing, vol. 25, no. 5, pp. 1559-1573, 2011.

[36] M. Nobahari, M. R. Ghasemi, and N. Shabakhty, "A fast and robust method for damage detection of truss structures," Applied Mathematical Modelling, vol. 68, pp. 368-382, 2019.

[37] J. Guo, D. Guan, and J. Zhao, "Structural damage identification based on the wavelet transform and improved particle swarm optimization algorithm," Advances in Civil Engineering, vol. 2020, Article ID 8869810, 19 pages, 2020.

[38] S. Loutridis, E. Douka, L. J. Hadjileontiadis, and A. Trochidis, "A two-dimensional wavelet transform for detection of cracks in plates," Engineering Structures, vol. 27, no. 9, pp. 1327-1338, 2005.

[39] M. Rucka, "Damage detection in beams using wavelet transform on higher vibration modes," Journal of Theoretical \& Applied Mechanics, vol. 49, pp. 399-417, 2011.

[40] M. Cao, M. Radzieński, W. Xu, and W. Ostachowicz, "Identification of multiple damage in beams based on robust curvature mode shapes," Mechanical Systems and Signal Processing, vol. 46, no. 2, pp. 468-480, 2014.

[41] W. Xu, M. Cao, W. Ostachowicz, M. Radzieński, and N. Xia, "Two-dimensional curvature mode shape method based on wavelets and Teager energy for damage detection in plates," Journal of Sound and Vibration, vol. 347, pp. 266-278, 2015.
[42] M. Rucka and K. Wilde, "Neuro-wavelet damage detection technique in beam, plate and shell structures with experimental validation," Journal of Theoretical and Applied Mechanics, vol. 48, no. 3, pp. 579-604, 2010.

[43] G. R. Irwin, "Plastic zone near a crack and fracture toughness," in Proceedings of the 7th Sagamore Research Conference on Mechanics \& Metals Behavior of Sheet Material, vol. 4, pp. 463-478, New York, NY, USA, January 1960.

[44] J. B. Ibso and H. Agerskov, "An analytical model for fatigue life prediction based on fracture mechanics and crack closure," Journal of Constructional Steel Research, vol. 37, no. 3, pp. 229-261, 1996.

[45] E. M. Morozov, "Evaluation of the critical sizes of surface cracks," Materials Science, vol. 42, no. 1, pp. 140-143, 2006.

[46] J. C. Newman and I. S. Raju, "An empirical stress-intensity factor equation for the surface crack," Engineering Fracture Mechanics, vol. 15, no. 1-2, pp. 185-192, 1981.

[47] J.-C. Hong, Y. Y. Kim, H. C. Lee, and Y. W. Lee, "Damage detection using the Lipschitz exponent estimated by the wavelet transform: applications to vibration modes of a beam," International Journal of Solids and Structures, vol. 39, no. 7, pp. 1803-1816, 2002.

[48] A. V. Ovanesova and L. E. Suárez, "Applications of wavelet transforms to damage detection in frame structures," Engineering Structures, vol. 26, no. 1, pp. 39-49, 2004.

[49] S. Zhong and S. O. Oyadiji, "Crack detection in simply supported beams using stationary wavelet transform of modal data," Structural Control and Health Monitoring, vol. 18, no. 2, pp. 169-190, 2011.

[50] F. Li, G. Meng, K. Kageyama, Z. Su, and L. Ye, "Optimal mother wavelet selection for Lamb wave analyses," Journal of Intelligent Material Systems \& Structures, vol. 20, pp. 11471161, 2009.

[51] M. M. R. Taha, A. Noureldin, J. L. Lucero, and T. J. Baca, "Wavelet transform for structural health monitoring: a compendium of uses and features," Structural Health Monitoring: An International Journal, vol. 5, no. 3, pp. 267-295, 2006. 